\title{
Influence of Bottom Topography on Vortex Stability
}

\author{
BOWEN ZHAO \\ Department of Geology and Geophysics, Yale University, New Haven, Connecticut \\ EMMA CHIEUSSE-GÉRARD \\ ENSTA ParisTech, Paris, France \\ GLENN FLIERL \\ Department of Earth, Atmospheric and Planetary Sciences, Massachusetts Institute of Technology, Cambridge, Massachusetts
}

(Manuscript received 24 February 2019, in final form 11 September 2019)

\begin{abstract}
The effects of topography on the linear stability of both barotropic vortices and two-layer, baroclinic vortices are examined by considering cylindrical topography and vortices with stepwise relative vorticity profiles in the quasigeostrophic approximation. Four vortex configurations are considered, classified by the number of relative vorticity steps in the horizontal and the number of layers in the vertical: barotropic onestep vortex (Rankine vortex), barotropic two-step vortex, and their two-layer, baroclinic counterparts with the vorticity steps in the upper layer. In the barotropic calculation, the vortex is destabilized by topography having an oppositely signed potential vorticity jump while stabilized by topography of same-signed jump, that is, anticyclones are destabilized by seamounts while stabilized by depressions. Further, topography of appropriate sign and magnitude can excite a mode- 1 instability for a two-step vortex, especially relevant for topographic encounters of an otherwise stable vortex. The baroclinic calculation is in general consistent with the barotropic calculation except that the growth rate weakens and, for a two-step vortex, becomes less sensitive to topography (sign and magnitude) as baroclinicity increases. The smaller growth rate for a baroclinic vortex is consistent with previous findings that vortices with sufficient baroclinic structure could cross the topography relatively easily. Nonlinear contour dynamics simulations are conducted to confirm the linear stability analysis and to describe the subsequent evolution.
\end{abstract}

\section{Introduction}

Vortices, also known as eddies or rings, are dominant features in the ocean. Oceanic bottom topography has been observed to influence vortices' trajectories and dissipation, altering their transport of momentum and tracers; a notable example is the Walvis Ridge blocking/destroying Agulhas rings, leading to local deposition of Indian Ocean water (Olson and Evans 1986; Schouten et al. 2000). Extensive effort has been put into understanding vortex-topography interactions. See LaCasce (1998) for a comprehensive review on early studies. Early studies with barotropic vortices using stability analysis or numerical/laboratory approaches revealed basic processes of this interaction, including radiation of topographic waves over bottom slopes and

\footnotetext{
Corresponding author: Bowen Zhao, bowen.zhao@yale.edu
}

cross-slope propagation via nonlinear self-advection (Carnevale et al. 1991; Wang 1992; Grimshaw et al. 1994a,b). Laboratory and numerical studies with barotropic vortices typically find that topography could strongly influence vortex trajectories and even lead to disintegration (van Geffen and Davies 2000; Zavala Sansón 2002). However, the ocean is stratified and numerical studies with baroclinic vortices found that vortices with sufficient baroclinic structure could cross the topography relatively easily with the topographic effect being mostly felt by the bottom layer (e.g., Smith and O'Brien 1983; Kamenkovich et al. 1996; LaCasce 1998; Beismann et al. 1999; Adams and Flierl 2010). This study examines the effect of local topography such as seamounts on both barotropic and baroclinic vortices via linear stability analysis.

Hart (1975a,b) examined the effect of topography on baroclinic stability of a circular current with a two-layer 
quasigeostrophic (QG) model. Yet his choice of upperlayer basic state as constant vorticity flow $\left(v_{1}^{(0)}=r\right.$ in his notation) is more appropriate for studying gyre circulations than vortices. Nycander and Lacasce (2004) used generalized variational principles and numerical simulations to study barotropic vortices over topography but concentrated on stable regimes with monotonic potential vorticity $(\mathrm{PV})$ profiles.

On the other hand, studies of 2D turbulence over topography such as those by Bretherton and Haidvogel (1976) show that the turbulence tends to make the PV more uniform, implying that there would be a negative correlation between the topography and the relative vorticity. This suggests that one would find anticyclonic (cyclonic) flow over elevated topography (depressions), which is confirmed by their numerical experiments. Similarly, studies examining homogeneous flow over topography tend to find anticyclones generated and trapped by seamounts (Huppert and Bryan 1976; Johnson 1978; Verron and Le Provost 1985).

In contrast, we are exploring situations in which a strong, preexisting vortex finds itself over topography; the basic state has strongly anomalous vorticity so that the total PV gradient does change sign either because of the topography or because the vortex is compact (i.e., strong shear). We approximate vortices following the approach of Flierl (1988), who examined linear as well as nonlinear stability of quasigeostrophic vortices in the absence of topography by approximating vortices as concentric columnar rings with stepwise PV profiles. This simple configuration allows us to explore a large portion of the parameter space by varying topographic height and the separation between vorticity steps. As we shall see, the potential for instability significantly alters the dynamics.

As noted in Flierl (1988), vortex instability can be interpreted as a result of phase-locking and subsequent mutual amplification among waves that exist on potential vorticity gradients. Topography also creates a potential vorticity gradient and hence supports topographic Rossby waves, which propagate with shallow water (high potential vorticity) on the right in the Northern Hemisphere or on the left in the Southern Hemisphere. Schmidt and Johnson (1997) further illustrate that horizontal shear destabilizes flow along ridges precisely through bringing two opposing topographic Rossby waves (on the two sides of a ridge) to be at relative rest. We will interpret our stability results following this phase-locking argument.

The energy source for perturbations to grow generally includes horizontal shear energy and available potential energy, referred to as barotropic conversion and barocinic conversion, respectively. Rayleigh shear-layer instability is a classical example for the former while the
Eady problem exemplifies the latter. In the barotropic vortex case, horizontal shear energy is the only possible energy source for instability whereas in the baroclinic vortex case both energy sources could contribute. We will examine the dominance of either energy source across the parameter space for a baroclinic vortex. It turns out that considering a rectilinear flow of piecewise uniform potential vorticity helps to illustrate the energetics in circular vortices, so we will also discuss that briefly.

Variations in the planetary vorticity $f$ (Coriolis parameter) are not expected to be essential for considering topography influence on vortex stability, so we adopt the $f$-plane approximation. We also limit the study to an inviscid, incompressible, QG flow and a cylindrical topography but remark that analyses of smooth topographies and vortices yield qualitatively similar results (see the appendix). The rest of the paper is organized as follows. We first present a general formulation of the stability problem in section 2 . In sections $3-6$, we consider four vortex configurations, classified by the number of relative vorticity jumps (not the number of potential vorticity jumps) and the number of vertical layers (i.e., one layer for barotropic vortex or two layers for baroclinic vortex). In sections 5 and 6 , we also present nonlinear contour dynamics simulations to confirm stability results. Last, we consider a rectilinear case (vortex radius being relaxed to infinity) to help illustrate on the energetics. We conclude with some discussion of oceanic applications in section 8 .

\section{Vortex instability}

We will approach the instability problem by casting it as an integral equation. Using piecewise constant potential vorticity profiles converts the integral into matrix operator so that we can easily find the growth rates. With the QG approximation, the equation of motion is the Lagragian conservation of quasigeostrophic potential vorticity $q$ :

$$
\frac{d q}{d t}=\frac{\partial q}{\partial t}+J(\psi, q)=0, \quad \text { with } \quad q=\mathscr{b} \psi+h,
$$

where $\psi$ is the streamfunction and $J(\cdot, \cdot)$ denotes the Jacobian. In the one-layer model

$$
\mathscr{L} \psi=\nabla^{2} \psi \quad \text { and } \quad h=\frac{f}{H} h_{t},
$$

where $H$ denotes the far-field fluid depth and $h_{t}$ denotes the topography elevation. In the two-layer model 


$$
\begin{aligned}
\mathscr{B} \psi= & {\left[\begin{array}{cc}
\nabla^{2}-\frac{f^{2}}{g^{\prime} H_{1}} & \frac{f^{2}}{g^{\prime} H_{1}} \\
\frac{f^{2}}{g^{\prime} H_{2}} & \nabla^{2}-\frac{f^{2}}{g^{\prime} H_{2}}
\end{array}\right]\left[\begin{array}{l}
\psi_{1} \\
\psi_{2}
\end{array}\right] \text { and } } \\
h= & {\left[\begin{array}{c}
0 \\
\frac{f}{H} h_{t}
\end{array}\right], }
\end{aligned}
$$

with $H_{1}$ and $H_{2}$ the upper- and lower-layer far-field depth, respectively. The equations will be nondimensionalized with the radius of the maximum vortex speed and $1 / f$; the topography will then appear as the ratio of the elevation to the mean lowerlayer depth.

The basic state $V(r)=(\partial / \partial r) \Psi$ has relative vorticity $\nabla^{2} \Psi=(1 / r)(\partial / \partial r) r V$ and an associated potential vorticity gradient $(\partial / \partial r) \mathscr{L} \Psi$. In the one-layer model

$$
\frac{\partial}{\partial r} \mathscr{L} \Psi=\frac{\partial}{\partial r} \nabla^{2} \Psi=\frac{1}{r} \frac{\partial}{\partial r} r \frac{\partial}{\partial r} V-\frac{1}{r^{2}} V \equiv L_{1} V
$$

where

$$
L_{m} \equiv \frac{1}{r} \frac{\partial}{\partial r} r \frac{\partial}{\partial r}-\frac{m^{2}}{r^{2}}
$$

is the Laplacian for azimuthal mode $m$. Note that topography is not involved in $V(r)$. Equivalent relations for the two-layer model will be given in section 4 .

Our basic state potential vorticity is

$$
Q(r)=\mathscr{C} \Psi+h,
$$

and the equation for the fluctuations is

$$
\frac{\partial}{\partial t} q^{\prime}+\frac{V}{r} q_{\theta}^{\prime}-\frac{1}{r} \psi_{\theta}^{\prime} Q_{r}+\frac{1}{r}\left(\psi_{r}^{\prime} q_{\theta}^{\prime}-\psi_{\theta}^{\prime} q_{r}^{\prime}\right)=0 .
$$

The stability problem

$$
\frac{\partial}{\partial t} q^{\prime}+\frac{V}{r} q_{\theta}^{\prime}-\frac{1}{r} \psi_{\theta}^{\prime} Q_{r}=0, \quad \mathscr{L} \psi^{\prime}=q^{\prime}
$$

can be written in terms of the displacements $\eta(r, \theta, t)$ of the PV contours $q^{\prime}=\eta Q_{r}$ as

$$
\frac{\partial}{\partial t} \eta+\frac{V}{r} \eta_{\theta}+\frac{1}{r} \psi_{\theta}^{\prime}=0, \quad \mathscr{L} \psi^{\prime}=-Q_{r} \eta
$$

or, for normal modes with $\eta$ and $\psi^{\prime}$ proportional to $\exp [\operatorname{im}(\theta-\omega t)]$,

$$
\frac{V}{r} \eta+\frac{1}{r} \psi^{\prime}=\omega \eta, \quad \mathscr{B} \psi^{\prime}=-Q_{r} \eta .
$$

Using the Green's function $G^{m}$ satisfying

$$
\mathscr{L} G^{m}\left(r \mid r^{\prime}\right) e^{\mathrm{Im} \theta}=\delta\left(r-r^{\prime}\right) e^{\mathrm{Im} \theta}
$$

gives

$$
\frac{V(r)}{r} \eta(r)-\frac{1}{r} \int d r^{\prime} G^{m}\left(r \mid r^{\prime}\right) Q_{r}\left(r^{\prime}\right) \eta\left(r^{\prime}\right)=\omega \eta(r) .
$$

As we shall describe in more detail below, for stepwise constant PV and cylindrical topography, $Q_{r}$ becomes a set of delta functions at $r=r_{i}$, and only the $\eta$ values at these radii matter; as a result, the integral equation becomes a matrix eigenvalue problem.

For baroclinic cases, $G^{m}\left(r_{i} \mid r_{j}\right)$ is more complex than the barotropic case, since it has to take into account the way jumps in different layers impact the movement of contours in both layers.

The basic state potential vorticity jumps arise from both the cylindrical topography and relative vorticity jumps. While the former is simply $h$, the latter will be denoted by $\Delta^{r_{j}}$ with $-\left[(\partial / \partial r) Q\left(r_{j}\right)\right]=\Delta^{r_{j}} \delta\left(r-r_{j}\right)$ with $\Delta^{r_{j}}$ being the PV anomaly inside the contour compared to that just outside. We will first consider a barotropic vortex with a one-step relative vorticity profile (barotropic Rankine vortex or BT-1) and its two-layer baroclinic counterpart, a two-layer Rankine-like vortex (BC-1) to illustrate the phase-locking interpretation of instability; then we will consider vortices with two-step vorticity profiles, in particular isolated vortices that approximate better real oceanic vortices: a barotropic two-step vortex (BT-2) and a two-layer, two-step vortex (BC-2). Figure 1 gives a sketch of all four vortex configurations. Their basic state potential vorticity jumps are summarized in Table 1 with subscripts referring to the layer while superscripts referring to the discontinuity location. Corresponding notations for potential vorticity contour displacements are summarized in Table 2.

\section{Barotropic Rankine vortex}

We begin with the simplest example, a barotropic Rankine vortex over topography, to illustrate the physics of the instability. This is somewhat analogous to the ridge problem studied by Schmidt and Johnson (1997). The otherwise stable vortex can be destabilized by topography which gives a potential vorticity jump of opposite sign from that associated with the vortex.

The relative vorticity of the unperturbed Rankine vortex is

$$
\zeta=\frac{1}{r} \frac{\partial}{\partial r} r V=2 v_{0} \mathscr{H}(1-r)
$$




\section{(a) Barotropic Rankin vortex}

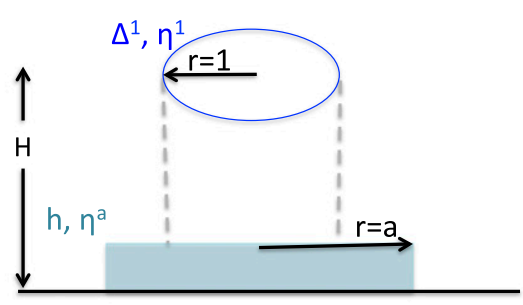

(b) two-layer, Rankin-like vortex

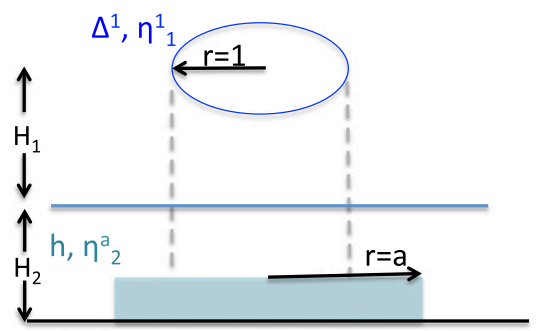

(c) Barotropic two-step vortex

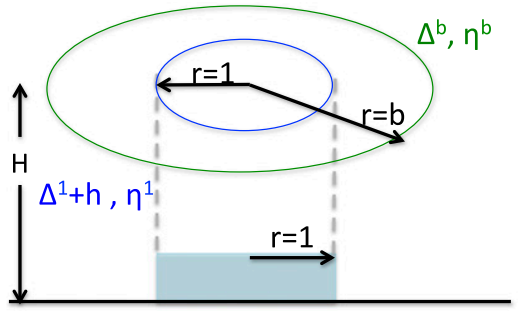

(d) two-layer, two-step vortex

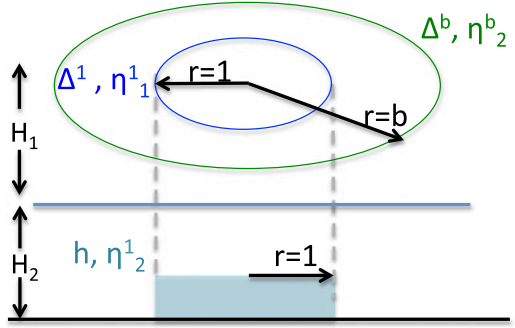

FIG. 1. Sketch of vortex configurations considered. (a) Barotropic Rankine vortex with one vorticity step at $r=1$ above a cylindrical topography with radius $a$. (c) Barotropic, two-step vortex with two vorticity steps at $r=1$ and $r=b$. The topography radius $a$ is fixed to be $r=1$ to reduce parameters. (b),(d) Two-layer versions of (a) and (c), respectively, with relative vorticity jumps in the upper layer and topography in the lower layer. The symbol $\Delta$ denotes relative vorticity jumps with superscripts denoting the discontinuity location. Topography-induced PV jump is denoted by $h$. The symbol $\eta$ denotes contour displacements with superscripts denoting the discontinuity location while subscripts denoting layers. Note that in the one-step vortex [(a) and (b)], we vary topography radius $r=a$ while in the two-step vortex we vary the outer contour radius $r=b$ with topography radius fixed at $r=1$. See Tables 1 and 2 for more on notations.

where $\mathscr{H}(\cdot)$ is the Heaviside step function. Here $v_{0}=1$ for cyclonic flow and -1 for the anticyclonic case. The basic state potential vorticity is

$$
Q=\zeta+h=2 v_{0} \mathscr{H}(1-r)+h \mathscr{H}(a-r) .
$$

The radius of the cylindrical seamount (divided by the radius of maximum velocity used to nondimensionalize the system) is $a$. Because the problem has steps in the potential vorticity, the gradient has singularities

$$
\begin{aligned}
Q_{r} & =-2 v_{0} \delta(r-1)-h \delta(r-a) \\
& \equiv-\Delta^{1} \delta(r-1)-\Delta^{a} \delta(r-a),
\end{aligned}
$$

where $\Delta^{r_{j}} \delta\left(r-r_{j}\right)=-Q_{r}\left(r_{j}\right)$ is the PV step from outside to inside. This structure implies we only need to consider the displacements at the set of radii $r_{j}$ where the

TABLE 1. Summary of basic state potential vorticity jumps for cases considered with subscripts referring to the layer while superscripts refer to the discontinuity location. We consider four vortex configurations, classified by the number of relative vorticity jumps in the horizontal and the number of layers in the vertical: 1) a barotropic one-step vortex (barotropic Rankine vortex or BT-1) with vorticity step at $r=1 ; 2$ ) a barotropic two-step vortex (BT-2) with vorticity steps at $r=1$ and $r=b ; 3$ ) a two-layer one-step vortex (BC-1); 4) a two-layer, two-step vortex (BC-2). The radius of cylindrical topography $a$ is variable in one-step vortices yet fixed at $a=1$ for two-step vortices, leading to collapsing of PV jumps \{i.e., for BT-2 one should add - $\left[(\partial / \partial r) Q_{1}(1)\right]$ and $-\left[(\partial / \partial r) Q_{1}(a)\right]$ to get a total PV jump of $\left.\Delta^{1}+h\right\}$. Note that, in two-layer vortices, we only consider upper-layer relative vorticity jumps so there is no need for labeling these with subscripts. See Fig. 1 for a sketch.

\begin{tabular}{lcccc}
\hline \hline Section & $-\left[(\partial / \partial r) Q_{1}(1)\right]$ & $-\left[(\partial / \partial r) Q_{1}(b)\right]$ & $-\left[(\partial / \partial r) Q_{1}(a)\right]$ & $-\left[(\partial / \partial r) Q_{2}(a)\right]$ \\
\hline 3: BT-1 & $\Delta^{1}$ & - & $h$ & - \\
4: BC-1 & $\Delta^{1}$ & - & - & $h$ \\
5: BT-2 & $\Delta^{1}$ & $\Delta^{b}$ & - & - \\
6: BC-2 & $\Delta^{1}$ & $\Delta^{b}$ & - & $h$ \\
\hline
\end{tabular}


TABLE 2. Summary of notations for potential vorticity contour displacements. Again, subscripts refer to the layer while superscripts refer to the discontinuity location. Note that subscripts are not needed for barotropic cases. Also note that setting $a=1$ in two-step vortices reduces the actual number of contours. See Fig. 1 for a sketch.

\begin{tabular}{lcccc}
\hline \hline Section & $\eta_{1}(1)$ & $\eta_{1}(b)$ & $\eta_{1}(a)$ & $\eta_{2}(a)$ \\
\hline 3: BT-1 & $\eta^{1}$ & - & $\eta^{a}$ & - \\
4: BC-1 & $\eta_{1}^{1}$ & - & - & $\eta_{2}^{a}$ \\
5: BT-2 & $\eta^{1}$ & $\eta^{b}$ & $\eta^{a}$ & - \\
6: BC-2 & $\eta_{1}^{1}$ & $\eta_{1}^{b}$ & - & $\eta_{2}^{a}$ \\
\hline
\end{tabular}

discontinuities occur; the integral in Eq. (1) is replaced by a sum, and the stability problem becomes

$$
\omega \eta\left(r_{i}\right)=\left[\frac{V\left(r_{i}\right)}{r_{i}} \delta_{i j}+\frac{1}{r_{i}} G^{m}\left(r_{i} \mid r_{j}\right) \Delta^{r_{j}}\right] \eta\left(r_{j}\right)
$$

(summation convention implied). For the barotropic mode, the Green's function is

$$
G^{m}\left(r \mid r^{\prime}\right)=-\frac{r^{\prime}}{2 m} \begin{cases}\left(r / r^{\prime}\right)^{m}, & r<r^{\prime} \\ K\left(r^{\prime} / r\right)^{m}, & r>r^{\prime}\end{cases}
$$

Substituting the factors for $Q_{r}$ (or, equivalently $\Delta^{1}=$ $2 v_{0}$ and $\left.\Delta^{a}=h\right), V$, and $G^{m}$ gives

$$
\mathbf{M}\left[\begin{array}{c}
\eta^{1} \\
\eta^{a}
\end{array}\right]=\omega\left[\begin{array}{l}
\eta^{1} \\
\eta^{a}
\end{array}\right],
$$

where

$$
\begin{aligned}
& \mathbf{M}(a \geq 1)=\left[\begin{array}{cc}
v_{0}-\frac{v_{0}}{m} & -\frac{a^{1-m} h}{2 m} \\
-\frac{v_{0} a^{-m-1}}{m} & \frac{v_{0}}{a^{2}}-\frac{h}{2 m}
\end{array}\right] ; \\
& \mathbf{M}(a<1)=\left[\begin{array}{cc}
v_{0}-\frac{v_{0}}{m} & -\frac{a^{1+m} h}{2 m} \\
-\frac{v_{0} a^{m-1}}{m} & \frac{v_{0}}{a^{2}}-\frac{h}{2 m}
\end{array}\right] .
\end{aligned}
$$

Note that matrix $\mathbf{M}$ changes as topography radius $a$ crosses 1 due to the piecewise Green's function [Eq. (2)]. The necessary condition is that the product of the offdiagonal terms must be negative, $v_{0} h<0$, so that the flow around a seamount has to be anticyclonic to be unstable. Note that, in this study, growth rates are not changed when topography and the flow change sign simultaneously, owing to the parity symmetry of QG theory. Below we choose $v_{0}=1$ and examine topography of both signs. In effect, this allows us to portray the results for cyclones and anticyclones over seamounts (positive $h v_{0}$ and negative $h v_{0}$ ) simultaneously. Calculation of the growth rates for the different modes shows that the vortex is indeed destabilized by the topography, with the $m=2,3,4, \ldots$ modes appearing in different ranges of the radius (Fig. 2). For $m=1$, the square of the trace is always greater than 4 times the determinant, so that mode is stable.

The physics leading to growth follows the usual pattern of mutually amplifying waves that are phase-locked. The necessary condition $v_{0} h<0$ provide the possibility that waves on the vortex contour can amplify the topographic waves and vice versa (the Rayleigh criterion). As in Schmidt and Johnson (1997), opposite PV gradients at $r=1$ and $r=a$ will lead to opposite directions of propagation of waves at these two PV discontinuities while a proper basic state velocity difference, $V(1)-V(a)$, can Doppler-shift them to phase-lock. This does not happen when $a<1$ or when $h<-(2 m-2)$ for $m \geq 2$ modes.

We can compare our results with some previous studies of barotropic vortices above topography. Nycander and Lacasce (2004) found analytically a large set of stable anticyclonic as well as cyclonic vortex solutions over a circular seamount but generally required a monotonic $\mathrm{PV}$ profile, which does not satisfy the necessary condition for instability (PV gradient changing sign). Moreover, growth rates calculated for Gaussian vorticity profiles and Gaussian-shaped topography as in Nycander and Lacasce (2004) but with the two fields having different radii yield similar conclusions as in the Rankine vortex case: in particular, only oppositely signed, larger scale topography gives instability (see the appendix). This further confirms that our stability result applies to smooth velocity profiles and smooth topography. Note that rotating shallow water models admit unstable modes for monotonic PV profiles owing to the radiation of inertia-gravity waves (i.e., radiative instability; Ford et al. 2000; Lahaye and Zeitlin 2016), which is however eliminated by our quasigeostrophy approximation. Due to small values of the Rossby number for oceanic eddies, we expect such growth rates to be very small and that radiation of inertia-gravity waves will not be important.

\section{Two-layer Rankine-like vortex}

We extend the barotropic Rankine vortex to a baroclinic one using a two-layer model by simply imposing a single potential vorticity jump in the upper layer and the cylindrical topography as the potential vorticity jump in the lower layer. The two-layer vortex has

$$
\begin{aligned}
& \frac{\partial}{\partial r} Q_{1}=-\Delta^{1} \delta(r-1), \\
& \frac{\partial}{\partial r} Q_{2}=-h \delta(r-a) .
\end{aligned}
$$



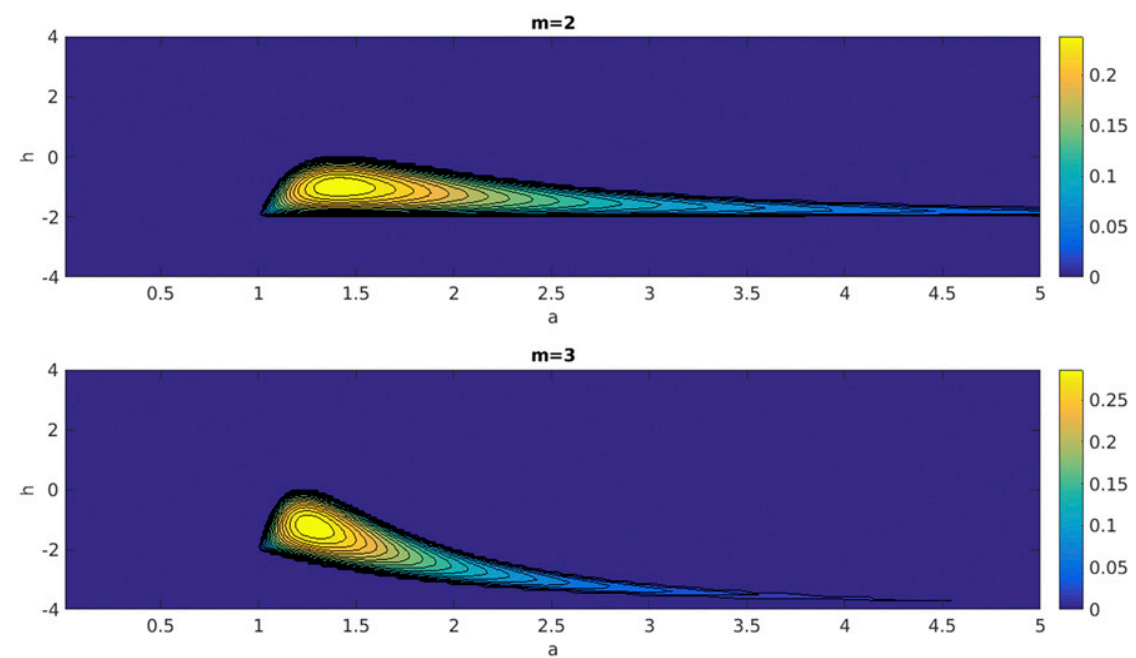

FIG. 2. Growth rate for the barotropic Rankine vortex as a function of topographic radius $a$ and topographic height $h$. Shown are (top) $m=2$ and (bottom) $m=3$. The $m=1$ mode remains stable. Note $v_{0}=1$ is chosen, and we present results for topography of both signs. Due to the parity symmetry of QG theory, this shows cyclones and anticyclones over seamounts (positive $h v_{0}$ and negative $h v_{0}$ ) simultaneously. The instability zone for $m \geq 2$ can be shown to vanish when $h<-(2 m-2)$.

The upper-layer basic velocity now also depends on the Rossby deformation radius $R_{d}$ defined as $1 / R_{d}^{2}=$ $\gamma^{2}=\left(f^{2} / g^{\prime} H_{1}\right)+\left(f^{2} / g^{\prime} H_{2}\right)=F_{1}+F_{2}$ in dimensional form, and the lower-layer velocity is nonzero. We use $H_{1}=H_{2}=H$ (which makes only a qualitative difference) so that $F_{1}=F_{2}=F$ and $\gamma^{2}=2 F$, giving one new parameter, the nondimensional deformation radius $1 / \gamma$. The two-layer $Q G$ equations can be added or subtracted to form the barotropic mode and the baroclinic mode:

$$
\begin{aligned}
& q_{\mathrm{BT}}=\nabla^{2} \psi_{\mathrm{BT}}+h(r), \quad \psi_{\mathrm{BT}}=\psi_{1}+\psi_{2} ; \\
& q_{\mathrm{BC}}=\nabla^{2} \psi_{\mathrm{BC}}-\gamma^{2} \psi_{\mathrm{BC}}-h(r), \quad \psi_{\mathrm{BC}}=\psi_{1}-\psi_{2} .
\end{aligned}
$$

The inversion relation for the basic state velocities can be written in the general form in terms of the vorticity jumps $\Delta_{\ell}\left(r_{j}\right)$ in layer $\ell$ at radius $r_{j}$ :

$$
V_{\ell}\left(r_{i}\right)=-G_{\ell n}^{1}\left(r_{i} \mid r_{j}\right) \Delta_{n}\left(r_{j}\right)
$$

summed over layer index $n$ and locations $j$. The Green's function matrix can be written in terms of the barotropic $G^{m}$ in Eq. (2) and the baroclinic mode Green's function $\mathscr{G}^{m}$ satisfying

$$
\left(L_{m}-\gamma^{2}\right) \mathscr{G}^{m}\left(r \mid r^{\prime}\right)=\delta\left(r-r^{\prime}\right),
$$

which gives

$$
\mathscr{G}^{m}\left(r \mid r^{\prime}\right)=-r^{\prime}\left\{\begin{array}{ll}
I_{m}(\gamma r) K_{m}\left(\gamma r^{\prime}\right), & r<r^{\prime} \\
I_{m}\left(\gamma r^{\prime}\right) K_{m}(\gamma r), & r>r^{\prime}
\end{array} .\right.
$$

Combining these by, in essence, decomposing a jump in one layer into the modes, finding the modal amplitudes from the two Green's functions, and reprojecting onto the layers gives (for the case $H_{1}=H_{2}$ )

$$
\begin{aligned}
& G_{11}^{m}\left(r \mid r^{\prime}\right)=\frac{1}{2}\left[G^{m}\left(r \mid r^{\prime}\right)+\mathscr{G}^{m}\left(r \mid r^{\prime}\right)\right], \\
& G_{12}^{m}\left(r \mid r^{\prime}\right)=\frac{1}{2}\left[G^{m}\left(r \mid r^{\prime}\right)-\mathscr{G}^{m}\left(r \mid r^{\prime}\right)\right], \\
& G_{21}^{m}\left(r \mid r^{\prime}\right)=\frac{1}{2}\left[G^{m}\left(r \mid r^{\prime}\right)-\mathscr{G}^{m}\left(r \mid r^{\prime}\right)\right], \quad \text { and } \\
& G_{22}^{m}\left(r \mid r^{\prime}\right)=\frac{1}{2}\left[G^{m}\left(r \mid r^{\prime}\right)+\mathscr{S}^{m}\left(r \mid r^{\prime}\right)\right] .
\end{aligned}
$$

With a single upper-layer vorticity jump $\Delta^{1}$, we have

$$
V_{1}(1)=-G_{11}^{1}(1,1) \Delta^{1} \quad \text { and } \quad V_{2}(a)=-G_{21}^{1}(a, 1) \Delta^{1},
$$

and we choose $\Delta^{1}=-1 / G_{11}^{1}(1,1)$ so that $V_{1}(1)=1$. Our eigenvalue problem now has a matrix

$$
\mathbf{M}=\left[\begin{array}{cc}
G_{11}^{m}(1 \mid 1) \Delta^{1}+1 & G_{12}^{m}(1 \mid a) h \\
G_{21}^{m}(a \mid 1) \Delta^{1} / a & G_{22}^{m}(a \mid a) h / a+V_{2}(a) / a
\end{array}\right] .
$$

Calculations again show that depressions destabilize cyclones for $m=2,3,4, \ldots$ (Fig. 3) while mode- 1 remains stable. As $R_{d}$ increases, the instability zone shrinks while growth rate decreases. This indicates that 

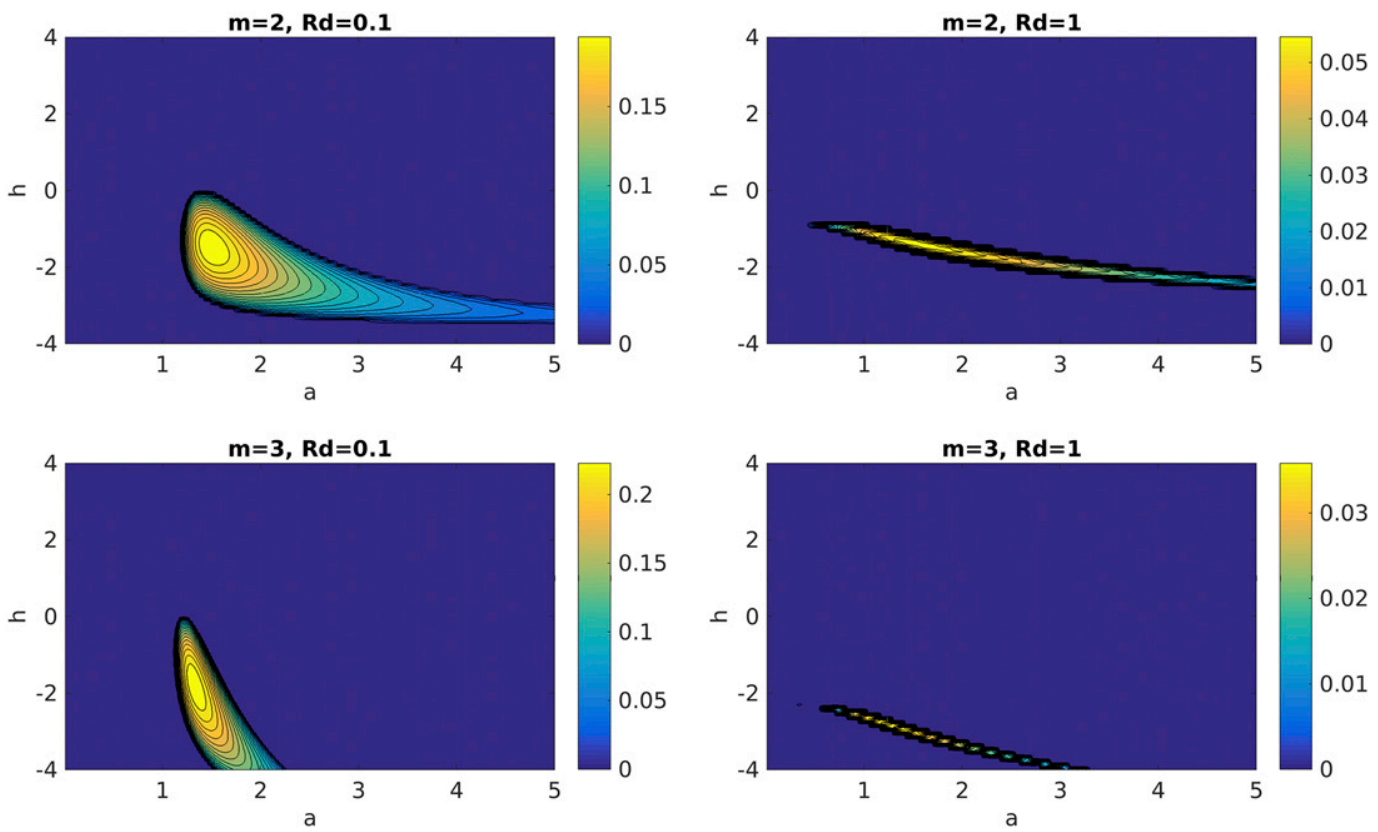

FIG. 3. Growth rate for the baroclinic Rankine-like vortex (BC-1 or one-step, two-layer vortex). Shown are growth rate for (top) $m=2$ and (bottom) $m=3$ modes, as a function of topographic $a$ and topography $h$. The $m=1$ mode remain stable. Shown are (left) $R_{d}=0.1$, and (right) $R_{d}=1$.

the effect of topography weakens as the layers gradually decouple, consistent with previous baroclinic results (e.g., Smith and O'Brien 1983; Kamenkovich et al. 1996; LaCasce 1998; Adams and Flierl 2010).

The simple correspondence between the baroclinic and barotropic one-step vortex follows from the necessary condition for instability that the PV gradient $Q_{r}$ has to change sign. For a vortex with one-step relative vorticity profile, the necessary condition can only be satisfied with oppositely signed topography. However, real oceanic vortices are compact, that is, the vortex velocity vanishes at finite distance. Thus, we next consider vortices with two-step relative vorticity profiles, the simplest possible model for a compact vortex.

\section{Two-step isolated barotropic vortex}

We first consider a barotropic two-step vortex. In particular, we examine only an isolated vortex with vanishing velocity in the far field, which better approximates oceanic eddies. We let the basic state velocity vanish at $r=b(b>1)$, which is also where the outer vorticity jump is located. Further, we set the radius of the cylindrical seamount $a=1$, that is, to coincide with the inner vorticity jump, to reduce the number of parameters (Fig. 1). In physical terms, we consider seamounts of comparable size to the vortex core. Note that this leads to a collapsing of $\eta^{1}$ and $\eta^{a}$ and that $(\partial / \partial r) Q(1)=(\partial / \partial r) Q(a)=\Delta^{1}+h$.
The basic state potential vorticity gradient becomes

$$
Q_{r}=-\left[\Delta^{1}+h\right] \delta(r-1)-\Delta^{b} \delta(r-b),
$$

where the $\Delta^{r_{j}}$ values are chosen to satisfy $V(1)=1$ and $V(b)=0$. These can be found from the inversion relation

$$
L_{1} V=\frac{\partial}{\partial r} \nabla^{2} \Psi=-\Delta^{r_{j}} \delta\left(r-r_{j}\right) \Rightarrow V\left(r_{i}\right)=-G^{1}\left(r_{i} \mid r_{j}\right) \Delta^{r_{j}},
$$

which gives $\Delta^{1}=2 v_{0} b^{2} /\left(b^{2}-1\right)$ and $\Delta^{b}=-2 v_{0} /\left(b^{2}-1\right)$. (We restore $v_{0}$ for the moment.) Note that the flow is determined by the relative vorticity jumps, not the potential vorticity jump associated with $h$. The matrix for the eigenvalue problem becomes

$$
\mathbf{M}=\left[\begin{array}{cc}
v_{0}-\frac{v_{0}}{m} \frac{b^{2}}{b^{2}-1}-\frac{h}{2 m} & \frac{v_{0}}{m} \frac{b^{1-m}}{b^{2}-1} \\
-\frac{v_{0}}{m} \frac{b^{1-m}}{b^{2}-1}-\frac{h b^{-m-1}}{2 m} & \frac{v_{0}}{m} \frac{1}{b^{2}-1}
\end{array}\right] .
$$

We will show that the $m=1$ mode can now be destabilized by the topography; the matrix

$$
\mathbf{M}=\left[\begin{array}{cc}
-\frac{v_{0}}{b^{2}-1}-\frac{h}{2} & \frac{v_{0}}{b^{2}-1} \\
-\frac{v_{0}}{b^{2}-1}-\frac{h}{2 b^{2}} & \frac{v_{0}}{b^{2}-1}
\end{array}\right]
$$



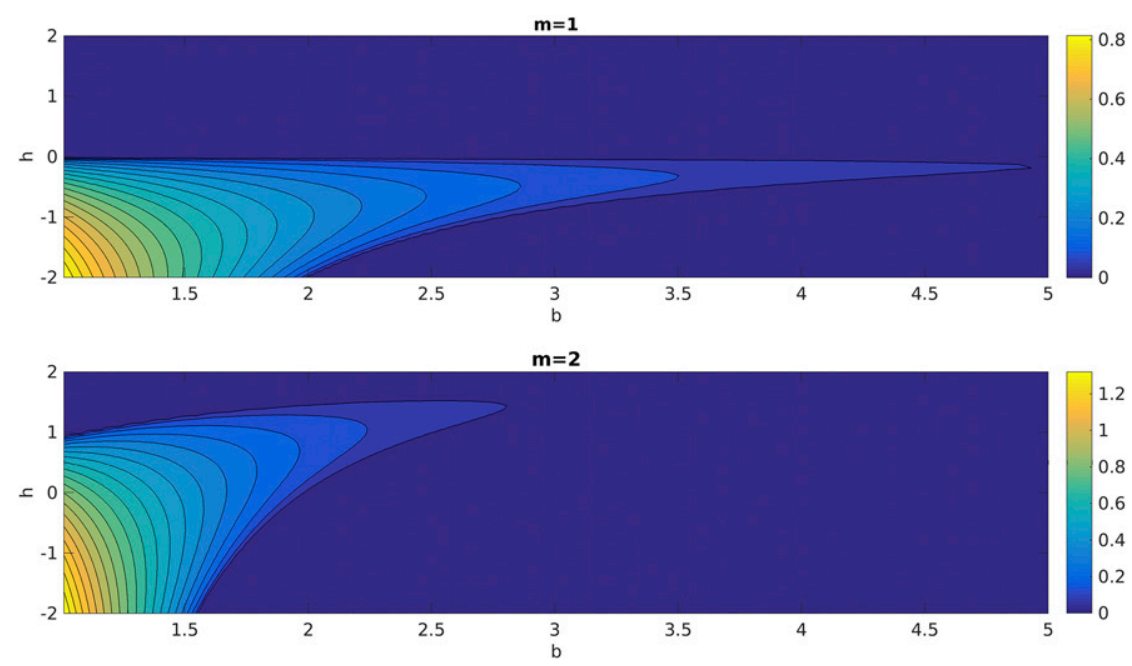

FIG. 4. Growth rate for the barotropic, two-step isolated vortex as a function of outer contour radius $b$ and topography height $h$. Shown are (top) $m=1$ and (bottom) $m=2$. Note $h$ ranges are different from Figs. 2 and 3.

is singular for $h=0$, and, moreover, $\omega=0$ is a degenerate eigenvalue. Biebuyck (1986) showed that when $V(b) \neq 0$, that is, the vortex is not isolated, the degenerate zero eigenvalue splits into two distinct eigenvalues: 0 corresponding to displacing the whole vortex trivially and $V(b) / b$ giving a rotation mode resulting from displacing the two contours relative to each other (i.e., having some projection on eigenmode $\left.\left[\begin{array}{ll}1 & -1\end{array}\right]^{\mathrm{T}}\right)$. The rotation mode turns into a translation mode $\{$ i.e., rotation radius $R \propto[b-V(b)] / V(b)$ goes to infinity) when $V(b)=0$ (Stern 1987; Stern and Radko 1998) and can be thought of as an algebraic instability. As we shall see, the fixed cylindrical boundary does not allow the translating mode, and, instead, it can now interact with the other mode to produce growth.

Topography will destabilize mode- 1 when

$$
h^{2}<-8 h \frac{v_{0}}{b^{2}}
$$

as shown in Fig. 4. This again requires $v_{0}$ and $h$ to be of opposite sign: a seamount destabilizes anticyclones while a depression destabilizes cyclones. Kizner et al. (2013) and Rabinovich et al. (2018) showed a similar $m=1$ instability for flow around an island. We can understand the mode- 1 dynamics better by noting that they represent the set of PV patches wobbling around the origin while remaining circular. To have a consistent rotation or growth, the velocity of each PV patch center should increase with its distance from the origin.

Reordering of the barotropic potential vorticity

$$
\begin{aligned}
\nabla^{2} \psi= & \left(\Delta^{1}+h\right) \mathscr{H}\left(1+\eta^{1}-r\right)+\Delta^{b} \mathscr{H}\left(b+\eta^{b}-r\right) \\
& -h \mathscr{H}(1-r)
\end{aligned}
$$

indicates that the velocity is determined by two PV patches whose boundaries can move (i.e., potential vorticity contours) and a PV patch whose boundary is fixed (i.e., the topography). A calculation as in Stern and Radko (1998) yields that if we shift the center of the inner active contour by $x_{1}$ and the outer one by $x_{b}$ we will get average northward motion of the inner PV patch as

$$
v_{1}=-\frac{h}{2} x_{1}+\frac{\Delta^{b}}{2}\left(x_{1}-x_{b}\right)
$$

with the first term from the topography and the second from the outer PV patch. Similarly, the effective northward velocity of the outer PV patch is

$$
v_{b}=-\frac{h}{2 b^{2}} x_{b}-\frac{\Delta^{1}+h}{2 b^{2}}\left(x_{1}-x_{b}\right)
$$

with the first term from the topography and the second term from the inner PV patch. Equating $v_{1} / x_{1}=$ $v_{b} / x_{b}=\omega$ leads to the same eigenvalue equation so we can understand the dispersion relation by examining the center movements, which is again the phaselocking reasoning. Note that $x_{1}$ and $x_{b}$ denote positions of centers of inner and outer PV patches; there is no restriction in their relative magnitude, that is, $x_{b}<x_{1}$ is as possible as $x_{b}>x_{1}$.

For $h=0$, the response to a displacement of the centers leads to equal velocities since $\Delta_{1}=-b^{2} \Delta_{b}$, the 

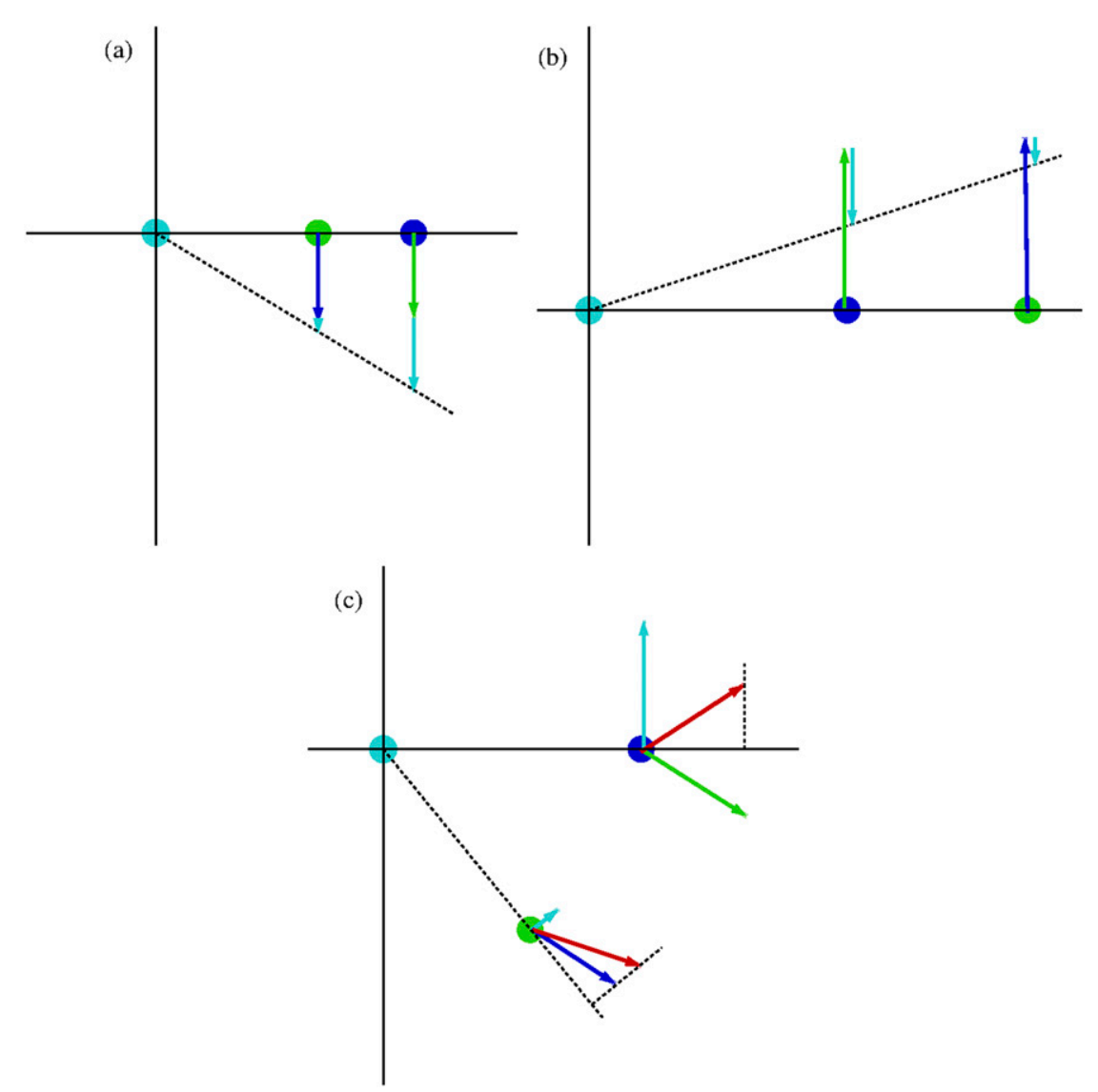

FIG. 5. (a)-(c) Sketch of induced velocities on PV patch centers. The inner disk center is represented by the blue solid circle while velocity induced by inner disk PV is represented by the blue arrow. The outer disk center is represented by the green solid circle while velocity induced by outer disk PV is represented by the green arrow. The topography center is represented by the cyan solid circle, and velocity induced by the topography is represented by the cyan arrow. In (a) $0<x_{b}<x_{1}$ and $y_{1}=y_{b}=0$. In (b) $0<x_{1}<x_{b}$ and $y_{1}=y_{b}=0$. In (c) $0<x_{b}<$ $x_{1}, y_{1}=0$ and $y_{b}<0$. The red arrows in (c) represent the net velocity including from the topography and the other PV patch. Note that the inner and outer PV patch centers (i.e., the blue and green dot) could swap position even though the outer contour radius $b$ could be much larger than 1 , since the center displacements are infinitesimal in linear theory.

translating state found by Stern (1987). With $h>0$, and $0<x_{b}<x_{1}$ (Fig. 5a), the contribution of the two contours to translation will be southward; the topography adds additional southward tendency to the inner patch and a weaker one to the closer-in center of the outer patch. These can be made consistent with a clockwise rotation $(\omega<0)$. If $h>0$ but $0<x_{1}<x_{b}$ (Fig. 5b), the interaction gives both centers a northward tendency, while the topography decreases that but less so for the further out $x_{b}$ center. Thus the mode rotates counterclockwise $(\omega>0)$. Instability occurs for $h<0$ when the vortices are no longer colinear with the center. Unstable modes correspond to a configuration such that both the outward and tangential velocities are proportional to the distances from the origin (see Fig. 5c for an example). The centers just rotate around the center of the topography and expand outwards.

Coming back to the linear stability of higher modes (Fig. 4): mode-2 could be unstable in the absence of topography provided that the vortex shear is strong enough (i.e., $b<2$ ) while higher modes require stronger shear for instability to exist [i.e., smaller $b$, not shown but see Flierl (1988)]. This is the classical barotropic instability: opposing vorticity jumps $\Delta^{1}$ and $\Delta^{b}$ support wave phase-locking. The topographic effect on mode-2 is similar to that for mode- 1 or the Rankine vortex with some subtlety: negative topography (seamounts for anticyclones) enhances the growth rate in general while stabilizing larger vortices (with $b \approx 2$ ); positive topography weakens the growth rate in general while it destabilizes larger vortices (with $b \approx 2$ ). 

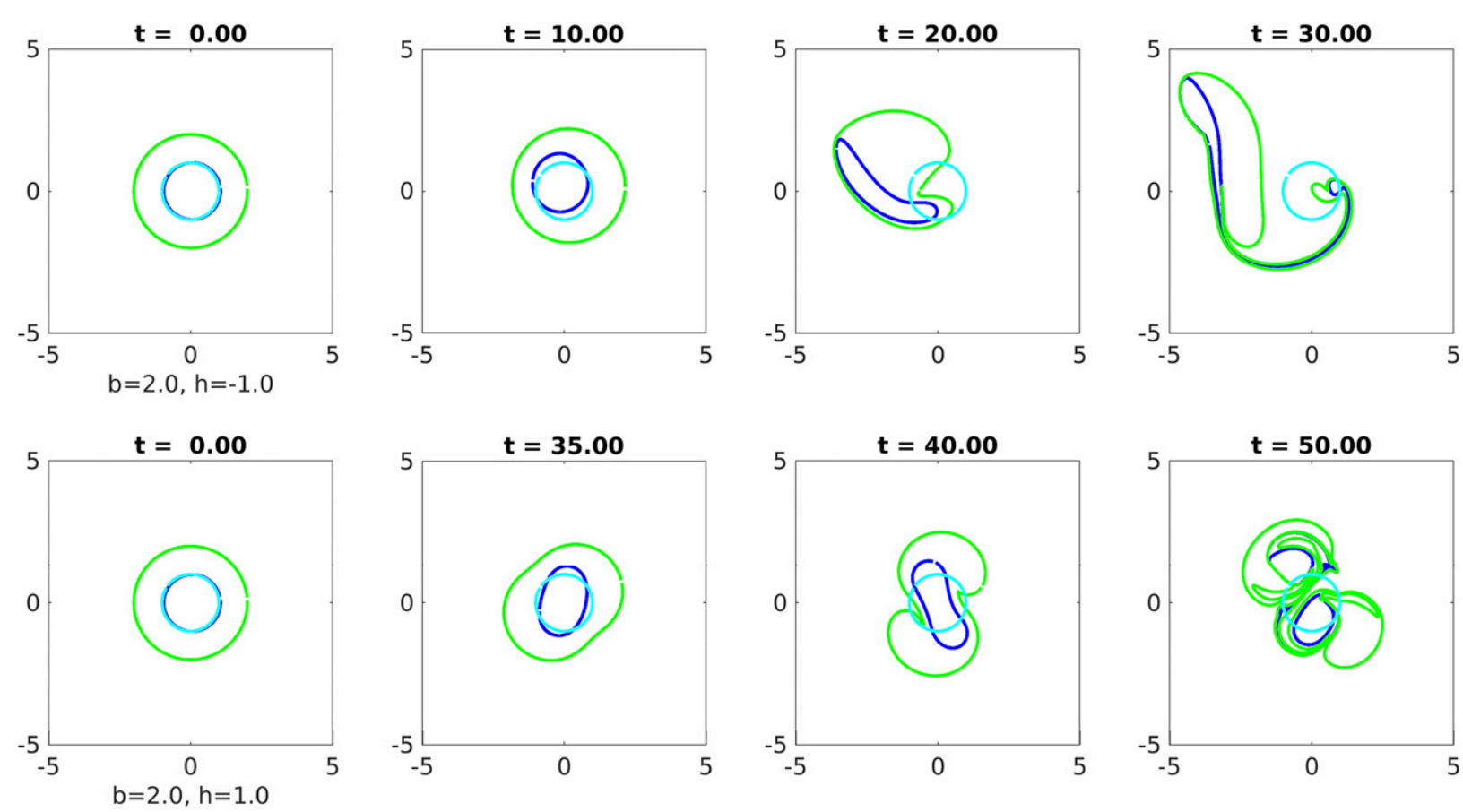

FIG. 6. Nonlinear contour dynamics simulation for the barotropic two-step vortex with (top) $b=2, h=-1$ and (bottom) $b=2, h=1$. The blue and green contours are associated with the relative vorticity jumps while the cyan contour denotes the topography, which is fixed. The blue contour is shifted laterally by 0.05 as the initial perturbation and hence instability builds up slowly in the bottom panel. Note we still keep $v_{0}=1$, and hence the top panels correspond to cyclones over depressions or anticyclones over seamounts (similar for the bottom panels).

\section{a. Nonlinear contour dynamics simulation}

We would like to confirm the linear stability results with nonlinear contour dynamics simulations (Zabusky et al. 1979; Pullin 1992), which employs a form similar to Eq. (3). That is, there are two active contours associated with the relative vorticity jumps, initially located at $r=1$ and $r=b$, and a fixed contour representing the topography. The contour dynamics routine does not use polar coordinates but instead tracks the contours as a set of Lagrangian points. For one experiment in Fig. 6, we choose $h=-1$ and $b=2$ such that linear stability analysis only renders mode- 1 unstable (we keep $v_{0}=1$ as noted above). The nonlinear simulation shows that mode- 1 is indeed destabilized first; the centers of the inner and outer contours increasingly shift relative to each other and the vortex moves further from the topography, though rotating around it also. Higher modes get excited later. In the other experiment in Fig. 6, we choose $h=1$ and $b=2$ such that linear stability analysis only renders mode- 2 unstable. It is seen that, in the nonlinear simulation, circular contours do deform into ellipses first. If we retain $h=1$ while decreasing $b$, we would observe that successive higher modes manifest themselves in the nonlinear simulation. In short, nonlinear simulation confirms linear stability results.
Thus, a compact anticyclone that would be stable in the flat-bottom case (having $b>2$ ) can suffer an $m=1$ instability and breakup over a seamount. While this is not strictly applicable with the problem of a stable ring encountering topography, since it does not speak to the changes that occur during the encounter, it seems consistent with previous Agulhas numerical studies showing that seamounts yield a strong influence on barotropic anticyclonic vortices (Zavala Sansón 2002; van Geffen and Davies 2000).

\section{Two-layer two-step vortex}

We now extend the two-step barotropic vortex to a twolayer version (topography radius $a=1$ still):

$$
\begin{aligned}
\frac{\partial}{\partial r} Q_{1} & =-\Delta^{1} \delta(r-1)-\Delta^{b} \delta(r-b), \\
\frac{\partial}{\partial r} Q_{2} & =-h \delta(r-1),
\end{aligned}
$$

with upper-layer potential vorticity jumps $\Delta^{1}$ and $\Delta^{b}$ such that upper-layer basic velocity satisfies $V_{1}(1)=1$ while $V_{1}(b)=0$.

The eigenvalue problem is 

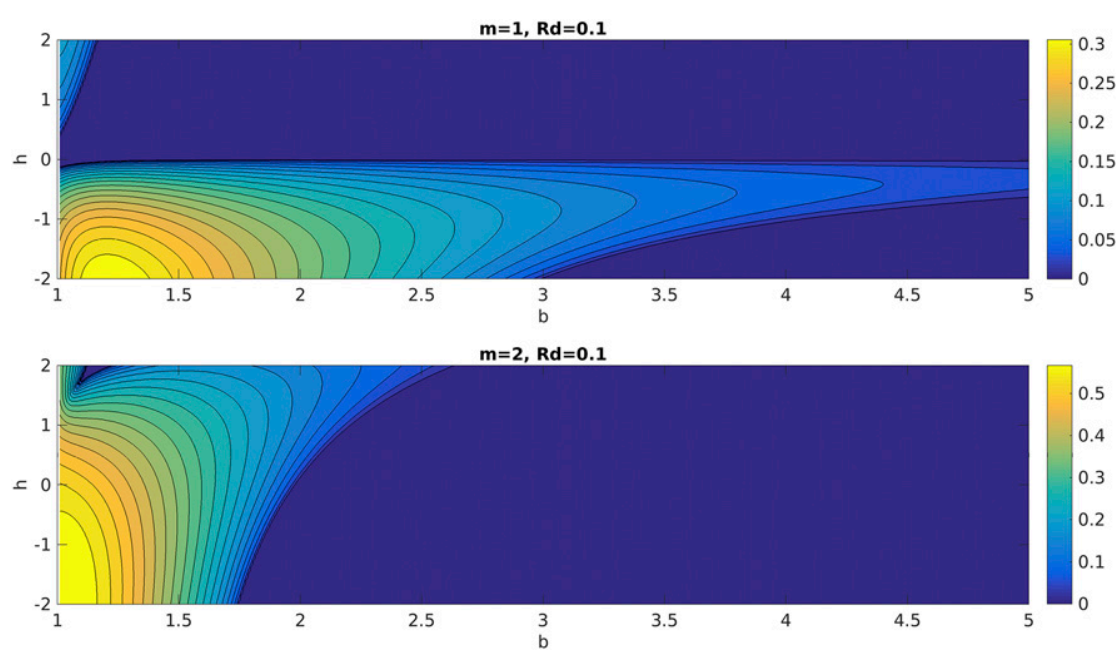

FIG. 7. Growth rate for the baroclinic two-step vortex (BC-2 or two-steps, two-layer vortex). Shown are growth rate for (top) $m=1$ and (bottom) $m=2$ modes, as a function of outer contour radius $b$ and topography height $h ; R_{d}$ is fixed at 0.1 .

$$
\mathbf{M} \boldsymbol{\eta}=\omega \boldsymbol{\eta},
$$

$$
\mathbf{M}=\left[\begin{array}{cc}
G_{11}^{m}(1 \mid 1) \Delta^{1}+1 & G_{11}^{m}(1 \mid b) \Delta^{b} \\
G_{11}^{m}(b \mid 1) \Delta^{1} / b & G_{11}^{m}(b \mid b) \Delta^{b} / b \\
G_{21}^{m}(1 \mid 1) \Delta^{1} & G_{21}^{m}(1 \mid b) \Delta^{b}
\end{array}\right.
$$

where $V_{2}(1)=-G_{21}^{1}(1 \mid 1) \Delta^{1}-G_{21}^{1}(1 \mid b) \Delta^{b}$.

Growth rates at small $R_{d}$ are very similar to the barotropic calculation (Fig. 7): mode-1 is stable with flat bottom (which follows easily from the $\mathbf{M}$ matrix) and can be destabilized by negative topography (seamounts for cyclones); mode-2 is in general destabilized by negative topography while stabilized by positive topography; higher modes are similar to mode-2, with the instability zone successively shrinking toward smaller $b$ (stronger shear). This similarity results from the strong coupling between two layers at small $R_{d}$ : the basic state flows $V_{1}(r)$ and $V_{2}(r)$ are quite similar. Since the topography is normalized here by the lower-layer depth instead of the total fluid depth, comparisons with the barotropic case in Fig. 4 should be made with the Fig. 7 value of $h$ reduced by a factor of 2 .

As $R_{d}$ increases further, the weak growth rate becomes less sensitive to the topography's sign as well as magnitude (Fig. 8); however it remains very small. Higher mode instabilities at large $R_{d}$ are again similar to mode-2 except for a successive shrinking toward smaller $b$. For $m=2$, also note the narrow instability tongue extending toward larger $b$ in the negative with the matrix, written in terms of the $G_{\ell n}^{m}$, being

$$
\left.\begin{array}{c}
G_{12}^{m}(1 \mid 1) h \\
G_{12}^{m}(b \mid 1) h / b \\
G_{22}^{m}(1 \mid 1) h+V_{2}(1)
\end{array}\right], \quad \boldsymbol{\eta}=\left[\begin{array}{c}
\eta_{1}^{1} \\
\eta_{1}^{b} \\
\eta_{2}^{1}
\end{array}\right],
$$

$h$ plane (clear in Fig. 8c but also in Fig. 8d after zoomin), which is reminiscent of the two-layer one-step vortex. This narrow tongue also persists for higher modes and will be discussed further below.

In short, the topographic effect weakens for two-step vortices as baroclinicity increases, similar to one-step vortex results. On the other hand, depressions and seamounts exert almost similar impacts when a two-step vortex is sufficiently baroclinic $\left(R_{d} \geq 1\right)$. This is not surprising for $m \geq 2$ modes: note that $m \geq 2$ modes are unstable with flat bottom provided that shear is strong $(b<2)$; thus the growth rate being insensitive to topography simply implies that topography has little effect. However, this insensitivity to topography sign and magnitude is surprising for $m=1$ mode given that $m=1$ mode is stable when $h=0$ (i.e., flat bottom). We will investigate this feature below with asymptotic analyses.

\section{a. Weak coupling limit}

To explore some of the less intuitive aspects of the stability diagrams for $m=1$ mode, we consider the limits of small and large $F$ (e.g., large and small $R_{d}$ compared to the vortex and topography radii). 

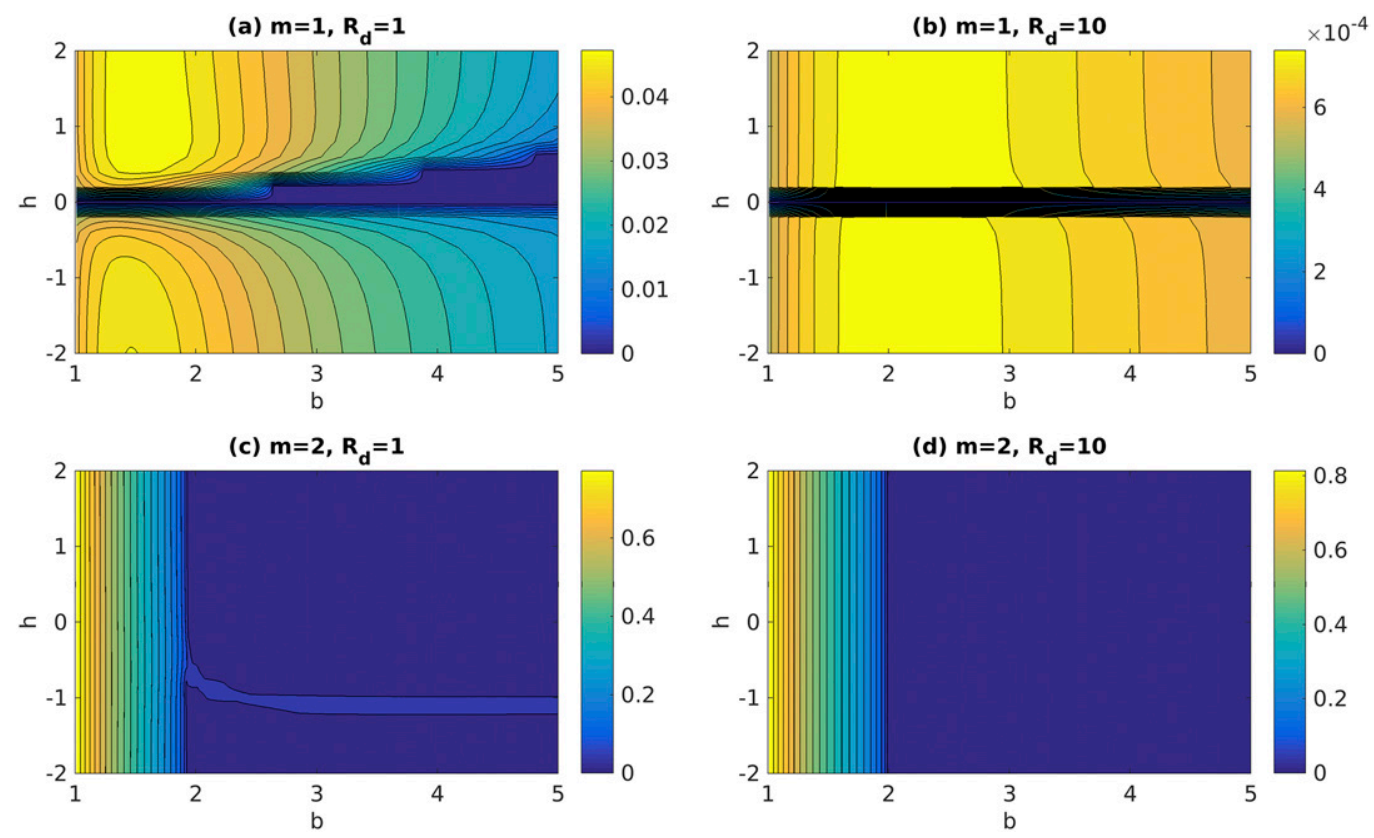

FIG. 8. As in Fig. 7, but for (left) $R_{d}=1$ and (right) $R_{d}=10$.

To explain the aforementioned insensitivity of growth rate to topography at large $R_{d}$, we employ a perturbation method with $F=(1 / 2)\left(1 / R_{d}^{2}\right)$ as the expansion parameter.

As $F \rightarrow 0$ or $R_{d} \rightarrow \infty$, the two layers become nearly independent, and we are left with two uncoupled, barotropic layers. At zeroth order, for mode-1,

$$
\mathbf{M}_{0}=\left[\begin{array}{ccc}
\frac{1}{1-b^{2}} & -\frac{1}{1-b^{2}} & 0 \\
\frac{1}{1-b^{2}} & -\frac{1}{1-b^{2}} & 0 \\
0 & 0 & -\frac{h}{2}
\end{array}\right]
$$

One eigenvalue $-(h / 2)$ with corresponding eigenvector $\left[\begin{array}{lll}0 & 0 & 1\end{array}\right]^{\mathrm{T}}$ is associated with the topographic Rossby wave. The other eigenvalue 0 is the degenerate mode discussed above; its eigenvector $\left[\begin{array}{lll}1 & 1 & 0\end{array}\right]^{\mathrm{T}}$ corresponds to a trivial shift of the upper-layer field. As $F$ increases or $R_{d}$ decreases, the two layers couple weakly and we expect the eigenvalue 0 to split into a complex conjugate pair. The first-order correction for $\omega_{0}=0$ is

$$
\mathbf{M}_{0} \boldsymbol{\eta}_{1}+\mathbf{M}_{1} \boldsymbol{\eta}_{0}=\omega_{0} \boldsymbol{\eta}_{1}+\omega_{1} \boldsymbol{\eta}_{0}=\omega_{1} \boldsymbol{\eta}_{0}
$$

where $\mathbf{M}_{1}, \boldsymbol{\eta}_{1}$, and $\omega_{1}$ are first-order corrections to $\mathbf{M}_{0}, \boldsymbol{\eta}_{0}=\left[\begin{array}{ccc}1 & 1 & 0\end{array}\right]^{\mathrm{T}}$, and $\omega_{0}=0$, respectively. Its projection on the lower-layer contour component (i.e., the third row)

$$
-\frac{h}{2} \boldsymbol{\eta}_{1}(3)+\mathbf{M}_{1}(3,1)+\mathbf{M}_{1}(3,2)=0
$$

simply states that the wave on the lower-layer contour should remain stationary under the collective influence of topography and upper-layer potential vorticity anomalies resulting from displacing upper-layer contours in the form of $\boldsymbol{\eta}_{0}$. This allows phase-locking to happen among waves on the three contours. Note $h \boldsymbol{\eta}_{1}(3)$ is thus set to be a constant to $\mathscr{Q}(F)$; in other words, the lower-layer contour displacement of this mode adjusts its sign and magnitude (relative to the other two contour displacements) for different topography so that it remains stationary.

The second-order correction, projecting on the left eigenvector (i.e., the solvability condition)

$$
\begin{aligned}
& \omega_{1}^{2}-\left[\mathbf{M}_{1}(1,1)-\mathbf{M}_{1}(2,1)\right] \omega_{1} \\
& +\frac{1}{b^{2}-1} \boldsymbol{\eta}_{1}(3)\left[\mathbf{M}_{1}(1,3)-\mathbf{M}_{1}(2,3)\right]=0,
\end{aligned}
$$

indicates possibility of complex roots and hence unstable modes. Note that $\mathbf{M}_{1}(1,3)$ and $\mathbf{M}_{2}(2,3)$ are proportional to $h$ yet $h \boldsymbol{\eta}_{1}(3)$ is constrained to be a constant as discussed above. Therefore $\omega_{1}$ turns out to be independent of $h$, explaining the insensitivity to topography seen in Fig. 8. The calculated first-order correction to growth rate is compared with direct numerical calculations in Fig. 9. It is seen that the first-order correction yields good approximation for $R_{d}>1.5$, confirming the stability results. 


\section{b. Strong coupling limit}

When $F \rightarrow \infty$ or $R_{d} \rightarrow 0$, the two layers are strongly coupled and we should recover the barotropic vortex case (Fig. 4), despite the fact that the topography is in a different layer.

With the definition $\phi=\psi_{1}-\psi_{2}$, the QG potential vorticity equation can be written as

$$
\begin{aligned}
\nabla^{2} \psi_{2}+F \phi= & h \mathscr{H}\left(1+\eta_{2}^{1}-r\right)-h \mathscr{H}(1-r), \\
\nabla^{2} \phi-2 F \phi= & \Delta^{r_{j}} \mathscr{H}\left(r_{j}+\eta^{r_{j}}-r\right)-h \mathscr{H}\left(1+\eta_{2}^{1}-r\right) \\
& +h \mathscr{H}(1-r) .
\end{aligned}
$$

The perturbation field at lowest order is

$$
\begin{aligned}
-2 F \phi & =\Delta^{r_{j}} \eta^{r_{j}} \delta\left(r_{j}-r\right)-h \eta_{2}^{1} \delta(1-r), \\
\nabla^{2} \psi_{2} & =\frac{1}{2} \Delta^{r_{j}} \eta^{r_{j}} \delta\left(r_{j}-r\right)+\frac{1}{2} h \eta_{2}^{1} \delta(1-r) .
\end{aligned}
$$

At zeroth order,

$$
\mathbf{M}_{0}=\left[\begin{array}{ccr}
\frac{1}{1-b^{2}} & -\frac{1}{1-b^{2}} & -\frac{h}{4} \\
\frac{1}{1-b^{2}} & -\frac{1}{1-b^{2}} & -\frac{h}{4 b^{2}} \\
\frac{b^{2}}{1-b^{2}} & -\frac{1}{1-b^{2}} & 1-\frac{h}{4}
\end{array}\right]
$$

The eigenvalues are

$$
\omega_{1}=1, \quad \omega_{2,3}=\frac{-\frac{h}{4} \pm \sqrt{\left(\frac{h}{4}\right)^{2}+\frac{h}{b^{2}}}}{2} .
$$

The eigenvalue 1 with corresponding eigenvector $\left[\begin{array}{lll}-\left(h / \Delta^{1}\right) & 0 & 1\end{array}\right]^{\mathrm{T}}$, is associated with the tilting of the inner column (Flierl 1988). Eigenvalues $\omega_{2,3}$ will be a complex conjugate pair when $(h / 4)$ $\left[(h / 4) b^{2}+4\right]<0$, corresponding to an unstable mode. The corresponding eigenvector reveals that the inner column is displaced as a whole (i.e., $\eta_{1}^{1}=\eta_{2}^{1}$ ), which is how a barotropic vortex should behave.

\section{c. Nonlinear contour dynamics simulation}

Again, we will confirm stability results with twolayer nonlinear contour dynamics simulation (Polvani et al. 1988). Differently from the barotropic nonlinear simulation, there are now three active contours, two associated with the upper-layer potential vorticity jumps and one tracing the fluid initially over the topography in the lower layer, in addition to the fixed contour representing the topography. The need for this can be illustrated by writing the polar coordinate form of the full potential vorticity analogous to Eq. (3):

$$
\begin{aligned}
\nabla^{2} \psi_{1}-F\left(\psi_{1}-\psi_{2}\right)= & \Delta^{1} \mathscr{H}\left[1+\eta_{1}^{1}(\theta, t)-r\right] \\
& +\Delta^{b} \mathscr{H}\left[b+\eta_{1}^{b}(\theta, t)-r\right], \\
\nabla^{2} \psi_{2}-F\left(\psi_{2}-\psi_{1}\right)+ & h \mathscr{H}(1-r)=h \mathscr{H}\left(1+\eta_{2}^{1}(\theta, t)-r\right) .
\end{aligned}
$$

The term on the right-hand side of the lower-layer equation ensures that there are no relative vorticity jumps in the lower layer initially when $\eta_{2}^{1}=0$, consistent with the form of $V_{2}$ and $(\partial / \partial r) Q_{2}$. When fluid is forced onto or off the topography, it develops vorticity anomalies.

Nonlinear simulations of perturbation growth with $R_{d}=0.1$ are shown in Fig. 10 for a case with only mode- 1 linearly unstable and a case with only mode-2 linearly unstable. The simulations for $R_{d}=0.1$ are quite similar to the barotropic nonlinear simulations shown in Fig. 6: the upper- and lower-layer contours evolve nearly synchronously (the red and blue contours in Fig. 10) due to the strong coupling when $F$ is large. Nonlinear contour dynamics simulation for relatively large $R_{d}$ (Fig. 11) confirms the insensitivity of stability to topography. The nonlinear simulation reveals that the upper-layer perturbation is dominated by mode- 1 growth while it drags some lower-layer fluid along; in contrast to the Fig. 10 case with strong $m=1$ growth, the upper-layer contours remain quite circular although shifted relative to each other and to the topography. This nonlinear robustness is consistent with the previous numerical findings that a baroclinic vortex could cross bottom topography relatively easily (Kamenkovich et al. 1996; LaCasce 1998; Beismann et al. 1999; Adams and Flierl 2010, although these studies have rather different geometry with the topography being a ridge or a slope).

As noted above, a vortex that is stable before encountering any topography (i.e., satisfying $b>2$ ) is most relevant for the vortex-topography encounter problem. Then mode- 1 is almost the only mode that could be destabilized by topography. As documented in earlier studies (Olson 1980, 1991; Goni et al. 1997; McDonagh et al. 1999; Garzoli et al. 1999; van Aken et al. 2003; Wei et al. 2008), Agulhas rings and Gulf Stream rings typically have (near) solid-body rotation within a core and radially decaying velocity outward, comparable to our simple vortex configuration. Agulhas rings are observed to have radii of maximum velocity around $100 \mathrm{~km}$ while Gulf Stream rings are smaller in size, with radius of maximum velocity around $50 \mathrm{~km}$. The deformation radius in their vicinity is on the order of 

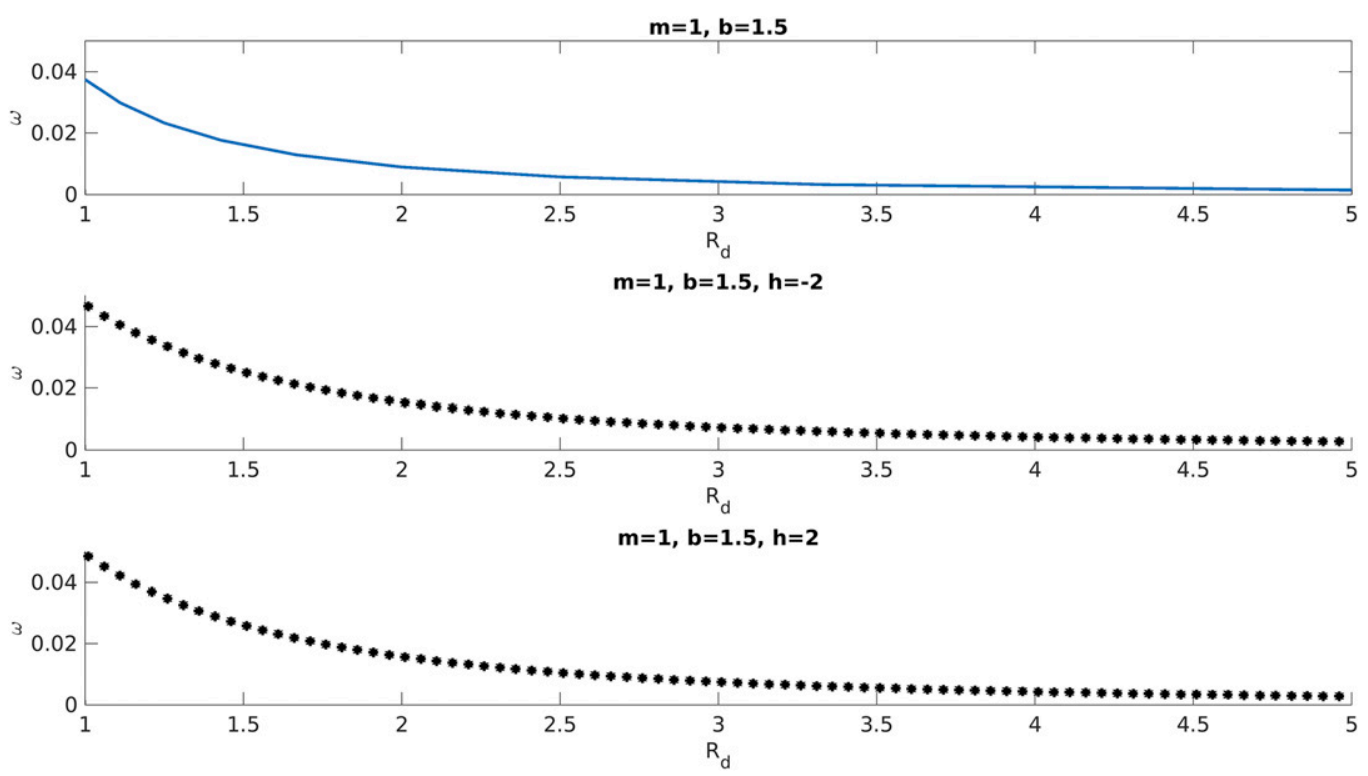

FIG. 9. Comparing the growth rate (the imaginary part of $\omega$ ) based on (top) the first-order correction and (middle),(bottom) that from direct numerical calculation. Note the $x$ axis starts from $R_{d}=1, h= \pm 2$ in the numerical calculation (the magnitude of $h$ changes little), and $b=1.5$ in all three panels.

$30-40 \mathrm{~km}$ depending on the geographic location so that both fall into our $R_{d}<1$ range (recall that $R_{d}$ is defined to be Rossby deformation radius normalized by the radius of maximum velocity). Thus, stability results and nonlinear simulations suggest that seamounts could exert weak to moderate destabilizing effect on baroclinic Agulhas rings and warm core Gulf Stream rings. In the Agulhas ring case, we might anticipate that bumps on the Walvis ridge could have a destabilizing effect. As there still remains asymmetry between negative topography and positive topography when $R_{d}<1$, we expect seamount destabilizing effect on cold core Gulf Stream rings to be weaker than that on warm core Gulf Stream rings.

\section{d. Energetics}

Since both barotropic conversion and baroclinic conversion are possible energy sources for perturbation growth in the two-layer, two-step vortex, we would like to examine the dominance of one or the other. The eddy kinetic energy (EKE) and eddy available potential energy (EAPE) growth in the QG two-layer model are governed by

$$
\begin{aligned}
\frac{1}{H} \frac{\partial}{\partial t} K^{\prime}= & \int V_{1} u_{1} \zeta_{1}+\int V_{2} u_{2} \zeta_{2}+\frac{f}{H} \int w\left(\psi_{1}-\psi_{2}\right), \\
\frac{1}{H} \frac{\partial}{\partial t} A^{\prime}= & \frac{F}{2} \int\left(V_{1}-V_{2}\right)\left(u_{1}+u_{2}\right)\left(\psi_{1}-\psi_{2}\right) \\
& -\frac{f}{H} \int w\left(\psi_{1}-\psi_{2}\right),
\end{aligned}
$$

while the total eddy energy growth is obtained by adding the above two equations,

$$
\frac{1}{H} \frac{\partial}{\partial t}\left(K^{\prime}+A^{\prime}\right)=\sum_{i} \int V_{i} u_{i} q_{i}
$$

Barotropic conversion is identified as the $\sum_{i} \int V_{i} u_{i} \zeta_{i}=$ $\sum_{i} \int V_{i} u_{i} q_{i}-(F / 2) \int\left(V_{1}-V_{2}\right)\left(u_{1}+u_{2}\right)\left(\psi_{1}-\psi_{2}\right)$ term, while baroclinic conversion refers to $(F / 2) \int\left(V_{1}-\right.$ $\left.V_{2}\right)\left(u_{1}+u_{2}\right)\left(\psi_{1}-\psi_{2}\right)$.

As shown in Figs. 12 and 13, barotropic conversion dominates over baroclinic conversion throughout the $\left\{h, b, R_{d}\right\}$ phase space except in the previously noted narrow tongue with negative $h$ (clear in Fig. $13 \mathrm{c}$ but also Fig. 13c after zoom-in), where baroclinic conversion dominates, although barotropic conversion still takes place. We have confirmed the general dominance of barotropic conversion with nonlinear calculations (not shown). That the baroclinic conversion dominant region is associated with negative topography is consistent with the traditional notion that baroclinic conversion happens when upper-layer and lower-layer mean potential vorticity gradients (jumps) are of opposite sign. As remarked above, this instability zone is reminiscent of the two-layer Rankinelike vortex, where baroclinic conversion dominates when the upper-layer and lower-layer potential vorticity jumps are close to each other $(b<1.25)$. The topographic potential vorticity jump is at $r=b$ for that case while at $r=1$ in the two-step vortex so these are not directly comparable. 

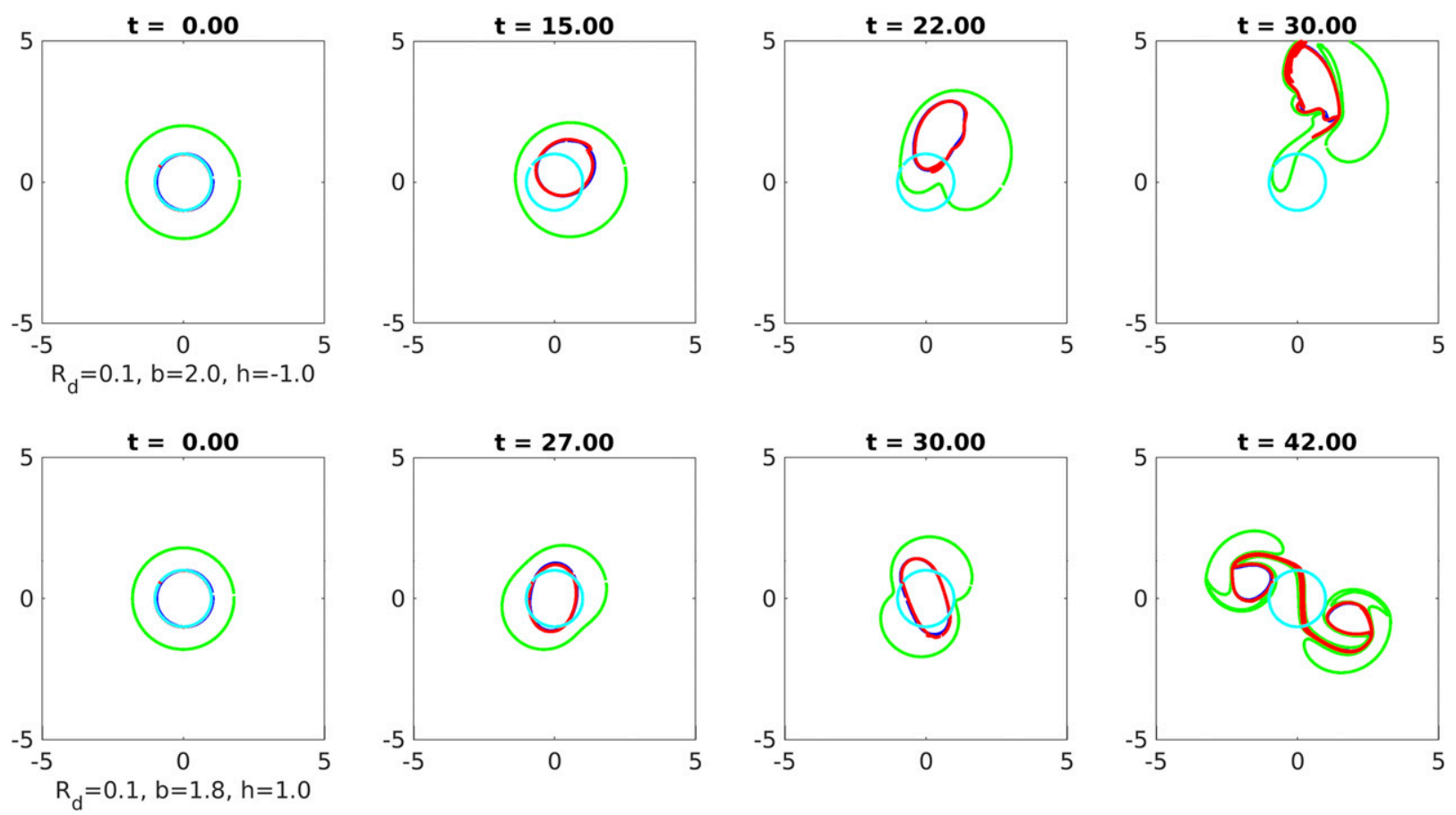

FIG. 10. Two-layer nonlinear contour dynamics simulation for $R_{d}=0.1$, with (top) $b=2.0, h=-1$ and (bottom) $b=1.8, h=1$. The blue and green contours are associated with the upper-layer potential vorticity jumps while the red contour is associated with the lower-layer potential vorticity jump. The cyan contour denotes the topography, which is fixed. The blue contour is shifted laterally by 0.05 as the initial perturbation.

We suspect that when the horizontal shear is reduced, the general dominance of barotropic conversion would disappear. To confirm this speculation, we next consider the piecewise uniform QG parallel flow (Meacham 1991; Schmidt and Johnson 1997), that is, relax the vortex radius to infinity.

\section{Rectilinear case}

The rectilinear system is set up in Cartesian coordinates with the $x$ axis along unperturbed contours. Let one upper-layer potential vorticity jump to be at $y=0$ and the second upper-layer potential vorticity jump to be at $y=1$. The previously cylindrical topography turns into a cliff at $y=0$. Lengths are now normalized with respect to the Rossby deformation radius $R_{d}$. Then the two-layer QG potential vorticity reads

$$
\begin{aligned}
& \nabla^{2} \psi_{1}-\frac{1}{2}\left(\psi_{1}-\psi_{2}\right)=\Delta^{0} \mathscr{H}\left(\eta_{1}^{0}-y\right)+\Delta^{1} \mathscr{H}\left(1+\eta_{1}^{1}-y\right), \\
& \nabla^{2} \psi_{2}+\frac{1}{2}\left(\psi_{1}-\psi_{2}\right)+h \mathscr{H}(-y)=h \mathscr{H}\left(\eta_{2}^{0}-y\right),
\end{aligned}
$$

where $\eta_{\ell}^{y_{j}}$ denotes potential vorticity contour displacements at $y_{j}$ in layer $\ell$. For this rectilinear system, we specify $\Delta^{y_{j}}$ instead of $V\left(y_{j}\right)$ : set $\Delta^{0}=1$ and vary $\Delta^{1}$ to consider different shear strengths. Figure 14 shows the growth rate for the $\Delta^{1}=0$ case and the $\Delta^{1}=-1$ case. For $\Delta^{1}=0$, there exists a narrow instability zone extending toward larger wavenumber as the topography magnitude increases. This instability zone is dominated by baroclinic conversion although barotropic conversion still takes place. The ratio of barotropic conversion and baroclinic conversion equals

$$
\frac{(\mu-k)(\mu+k+\gamma)}{\gamma^{2}}-1<1
$$

where $k$ is the wavenumber, $\gamma=1 / R_{d}$ and $\mu^{2}=k^{2}+\gamma^{2}$. For $\Delta^{1}=-1$, the baroclinic conversion dominant zone persists while a barotropic conversion dominant zone appears at smaller wavenumber. Comparing the two rectilinear cases clearly indicates that enhanced horizontal shear promotes dominance of barotropic conversion. The coexistence of a baroclinic conversion dominant regime and a barotropic conversion dominant regime is reminiscent of the vortex case.

\section{Conclusions}

We examined the effects of bottom topographic bumps or depressions on the linear stability of both barotropic vortices and two-layer, baroclinic vortices by considering cylindrical topography and vortices with stepwise vorticity profiles. We considered velocity profiles with one and with two discontinuities in 

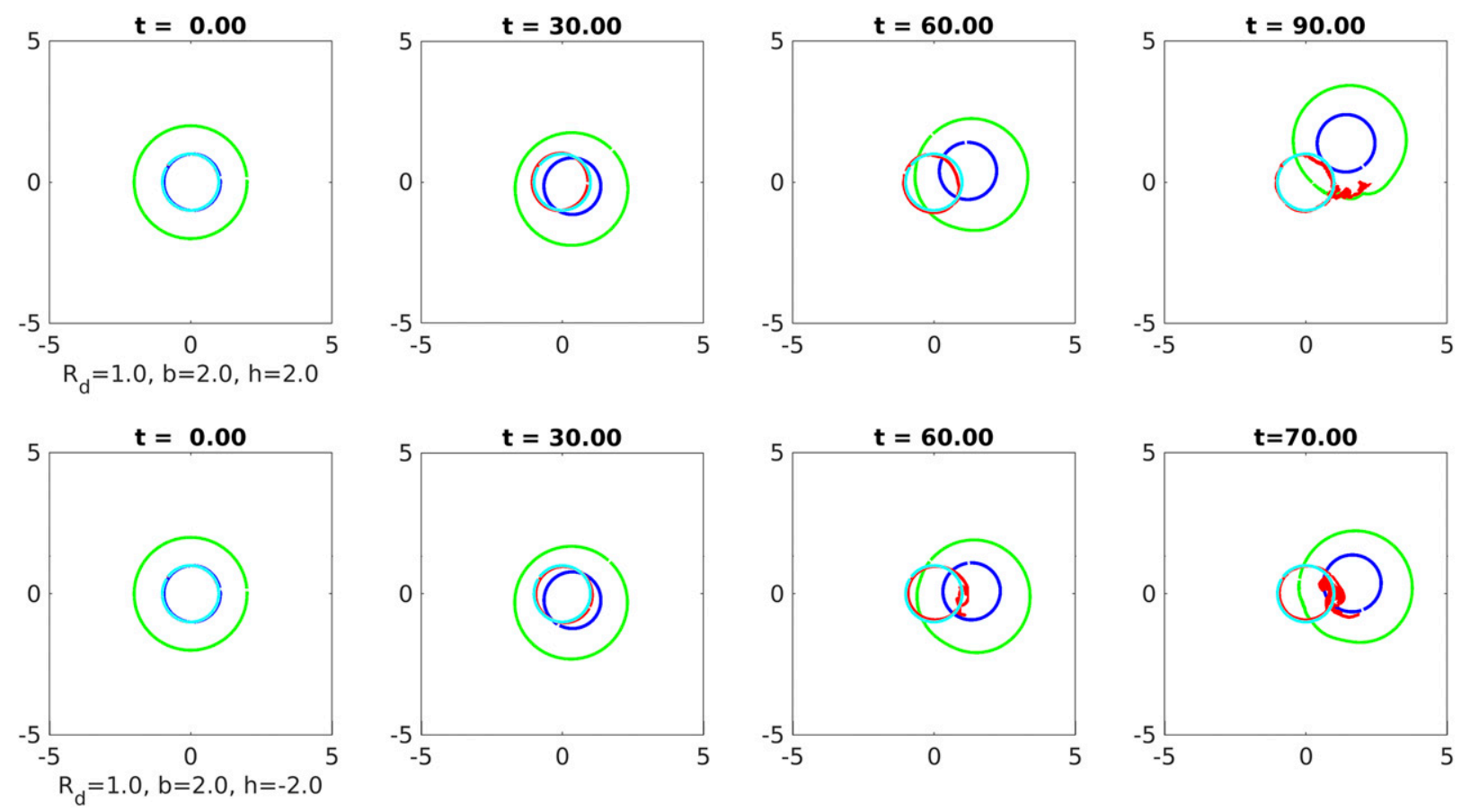

FIG. 11. Nonlinear contour dynamics simulation for $R_{d}=1, b=2.0$, with (top) $h=2.0$ and (bottom) $h=-2.0$. Only mode- 1 is unstable in both cases according to linear stability analysis. Note that the evolution for $h=2.0$ and $h=-2.0$ is very similar up to $t=60$.

the relative vorticity. For a vortex with one relative vorticity step, barotropic or baroclinic, only oppositely signed topography could excite instability. In physical terms, anticyclones get destabilized by seamounts and stabilized by depressions while the opposite is true for cyclones. This follows from the simple criterion that basic state PV gradient should change sign to allow waves supported at separate PV steps to mutually amplify. Baroclinicity only weakens growth rates and shrinks the instability zone for a one-step vortex (Fig. 3).

However, vortices with two-step vorticity profile (no net vorticity) approximate compact oceanic eddies better. For a barotropic two-step vortex, classical barotropic instability allows $m \geq 2$ modes to be unstable without topography for a sufficiently narrow outer band where the velocity falls to zero. Oppositely-signed topography (seamounts for anticyclones) generally destabilizes vortices while same-signed topography stabilizes them. Furthermore, oppositely signed topography can excite mode- 1 instability, which is especially relevant for vortextopography encounters. The baroclinic calculation for a two-step vortex is in general consistent with the barotropic calculation except that the growth rate weakens and that the topographic effect on strongly baroclinic vortices $\left(R_{d} \geq 1\right)$ is similar regardless of sign or magnitude. Our conclusions hold qualitatively for various layer depth ratios (see Fig. 15 for $H_{1} / H_{2}=1 / 5$ ).
Our baroclinic, two-step vortex with $R_{d} \geq 1$ (Rossby deformation radius greater than radius of maximum velocity) corresponds to shallow or surface intensified eddies discussed in previous studies. The fact that the instability mostly happens in the upper layer as a very slowly growing mode- 1 form is consistent with previous argument that a baroclinic vortex could cross the topography relatively easily (Smith and O'Brien 1983; Kamenkovich et al. 1996; LaCasce 1998; Beismann et al. 1999; Adams and Flierl 2010). In making connection between our simple two-step vortex model and real oceanic vortices, we noted that the part of parameter space with $b>2$ and $R_{d} \leq 1$ is most relevant. Thus stability results and nonlinear simulations support that seamounts with comparable radii could exert weak to moderate destabilizing effect on anticyclones, including Agulhas rings and warm core Gulf Stream rings.

A relevant yet different problem from this study is the topographically generated eddy problem. Anticyclones generated and maintained for long periods above seamounts have been reported in oceanographic observations (Richardson 1981; Gould et al. 1981), laboratory experiments (Zavala Sansón et al. 2012) and numerical simulations (Huppert and Bryan 1976; Verron and Le Provost 1985). The basic mechanism for such topographically generated eddies is the generation of vorticity anomalies as water column is squeezed or stretched during 

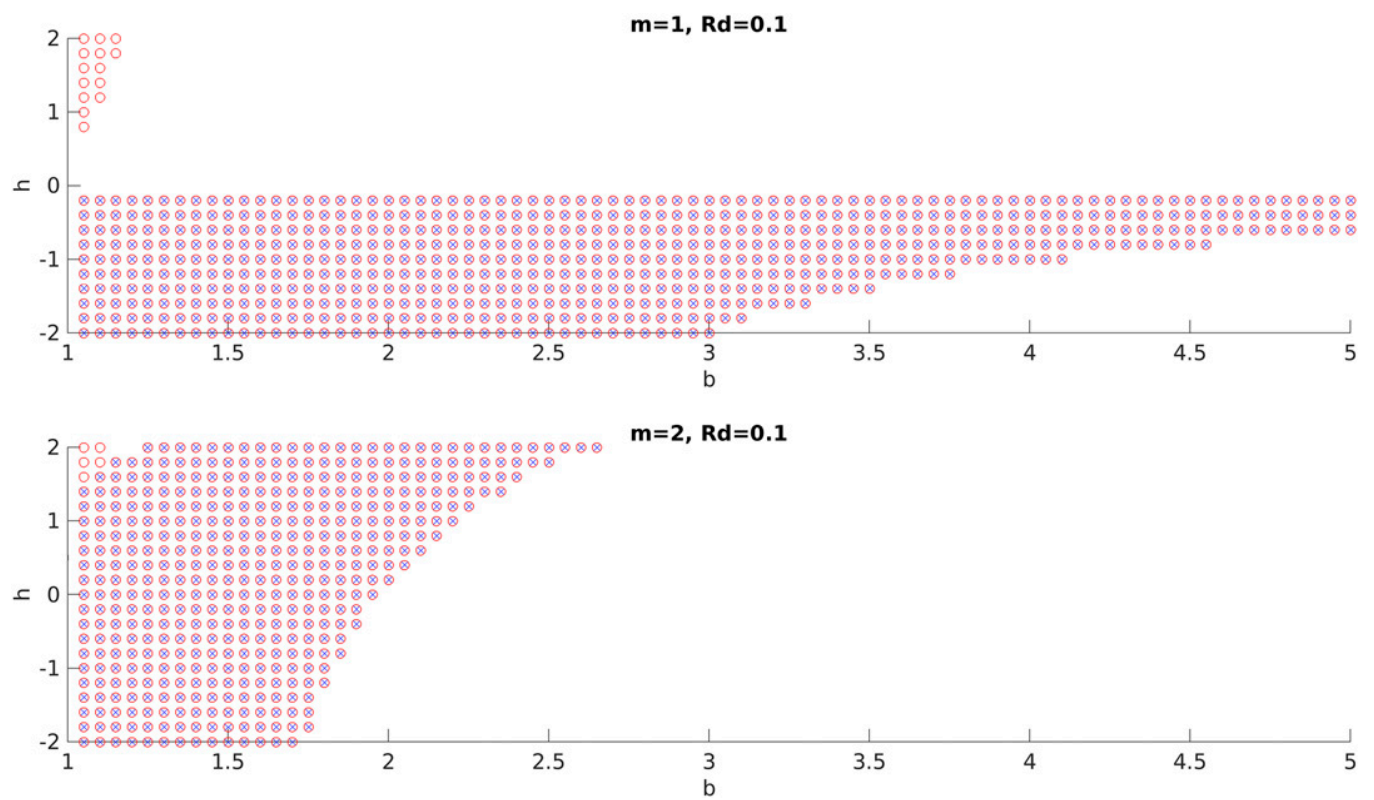

FIG. 12. Energetics as a function of outer contour radius $b$ and topography $h$. Shown are (top) $m=1$ and (bottom) $m=2 ; R_{d}=0.1$ is fixed. Circles are filled with blue crosses when baroclinic conversion happens while circles are painted red when barotropic conversion dominates baroclinic conversion.

advection across topography, conserving potential vorticity. These eddies tend to retain their original (i.e., ambient) PV such that the PV gradient should not experience a change in sign, which is necessarily a stable configuration according to our discussion. In contrast, the vortex considered in this study is not generated by topography but possesses a strong PV anomaly arising from its vorticity profile. The total PV gradient thus changes sign either because of the topography or because the vortex is compact. The apparent discrepancy between conclusions of this study and that of topographically generated eddy problem or $2 \mathrm{D}$ turbulence problem (see introduction) (a) $m=1, R_{d}=1$

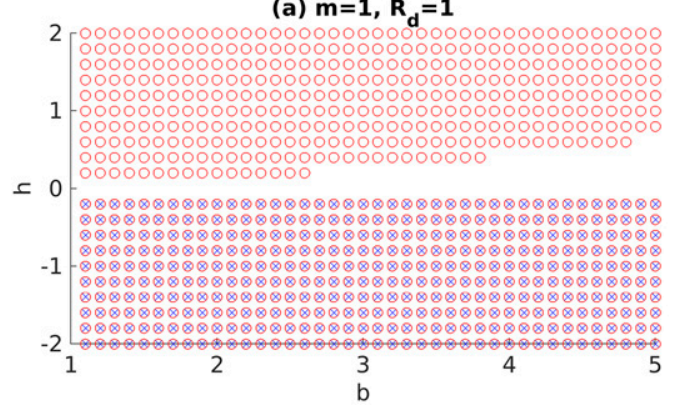

(c) $m=2, R_{d}=1$

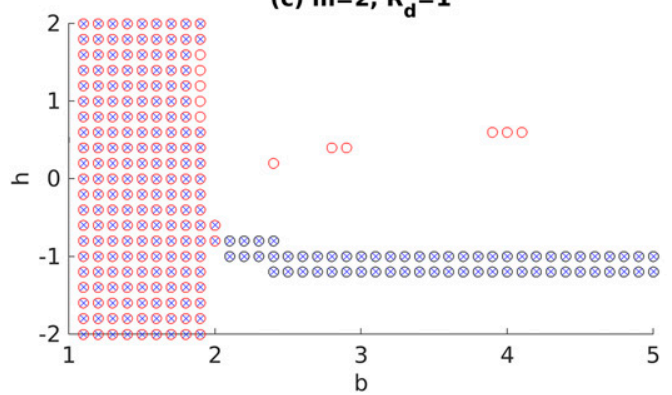

(b) $m=1, R_{d}=10$

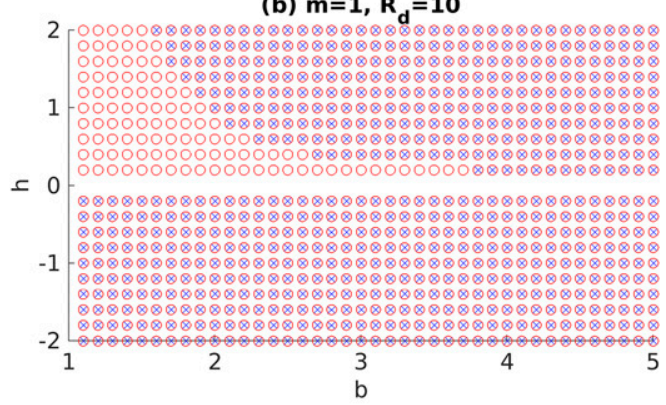

(d) $m=2, R_{d}=10$

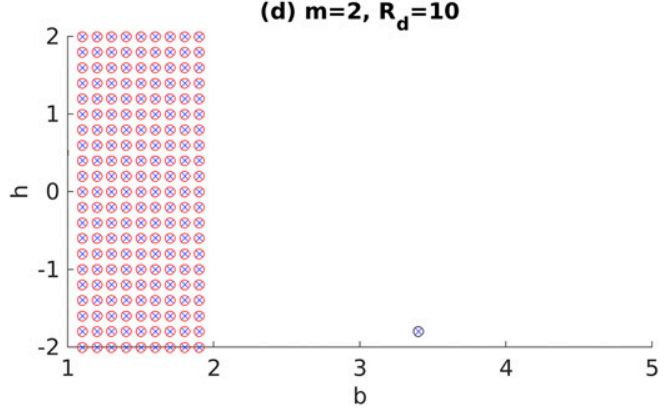

FIG. 13. As in Fig. 12, but for (left) $R_{d}=1$ and (right) $R_{d}=10$. 

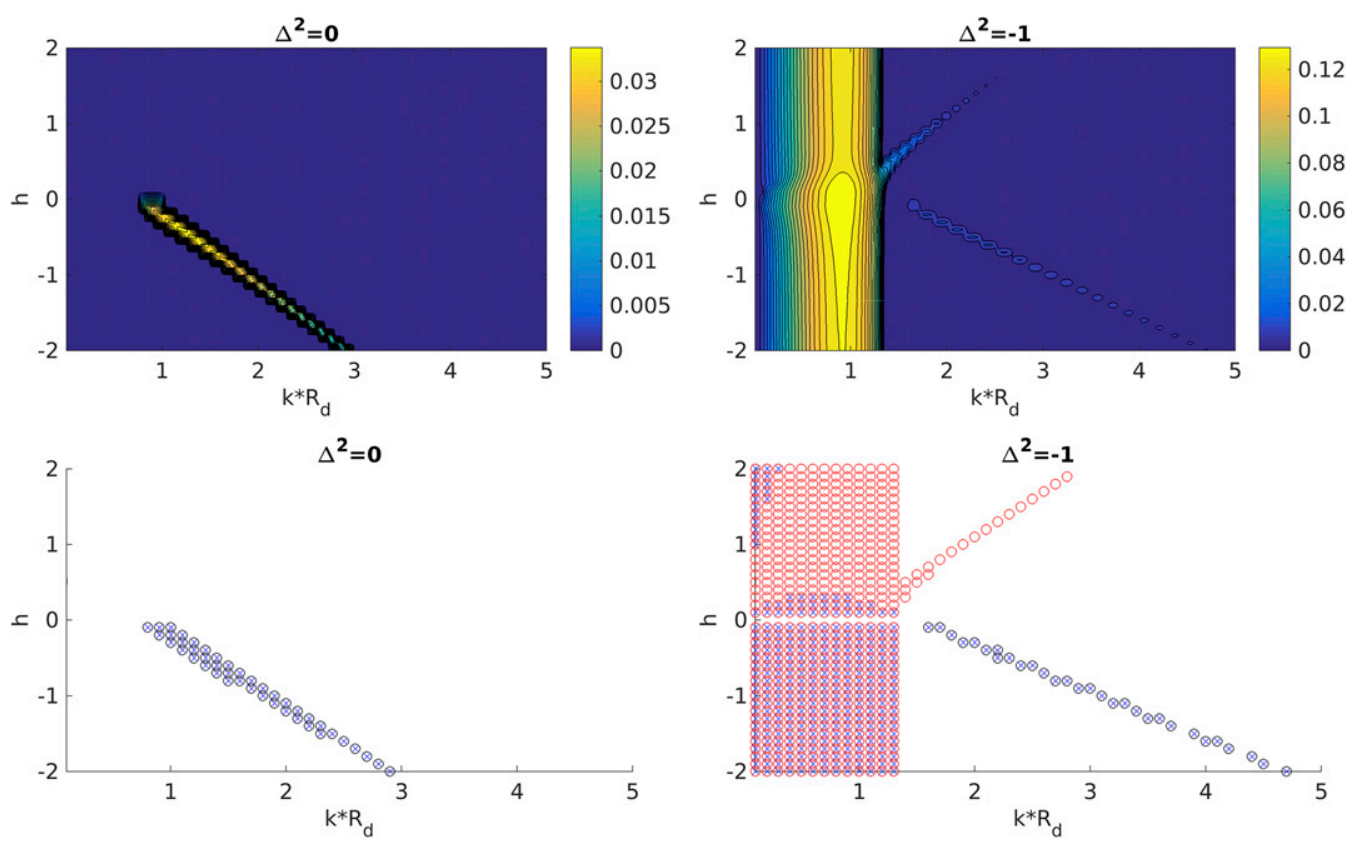

FIG. 14. (top) Growth rate and (bottom) energetics for two rectilinear cases: (left) $\Delta^{2}=0$ and (right) $\Delta^{2}=-1$. The $x$ axis is wavenumber normalized by $R_{d}$ while the $y$ axis is topography.

simply due to different basic state: the one in this study is strongly vortical while in previous studies (except Nycander and Lacasce 2004) had weak PV anomalies, either homogeneous or random.

We also note that linear stability analysis only considers infinitesimal perturbations. In realistic vortex colliding topography situation, the vortex center and
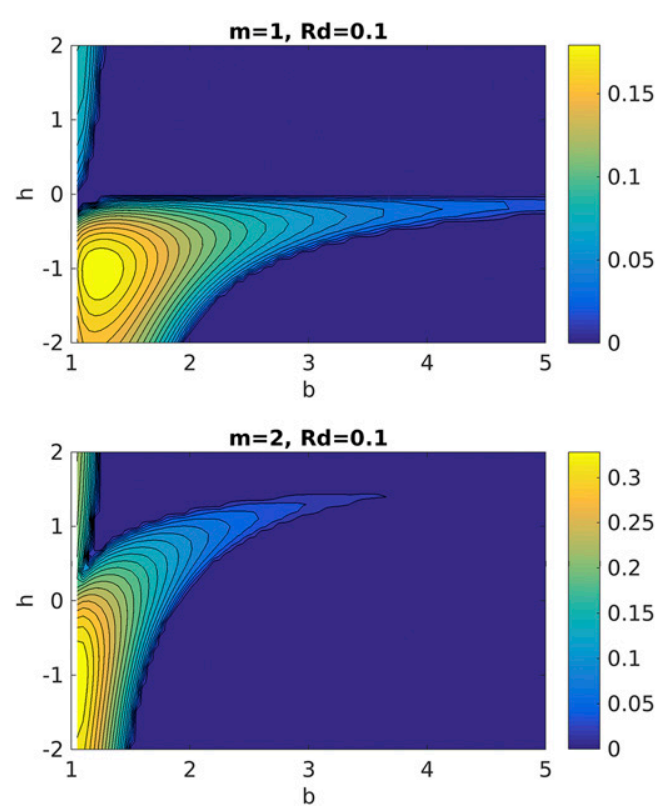

topography center would not be aligned, that is, a finite perturbation. We do not include time-dependent evolution when a linearly stable vortex approaching topography from far-field. For example, numerical simulations of Zavala Sansón et al. (2012) identified clockwise rotations for cyclones approaching a seamount (which are linear stable according to our results). Nevertheless, we expect

FIG. 15. Growth rate for $H_{1} / H_{2}=1 / 5$. 

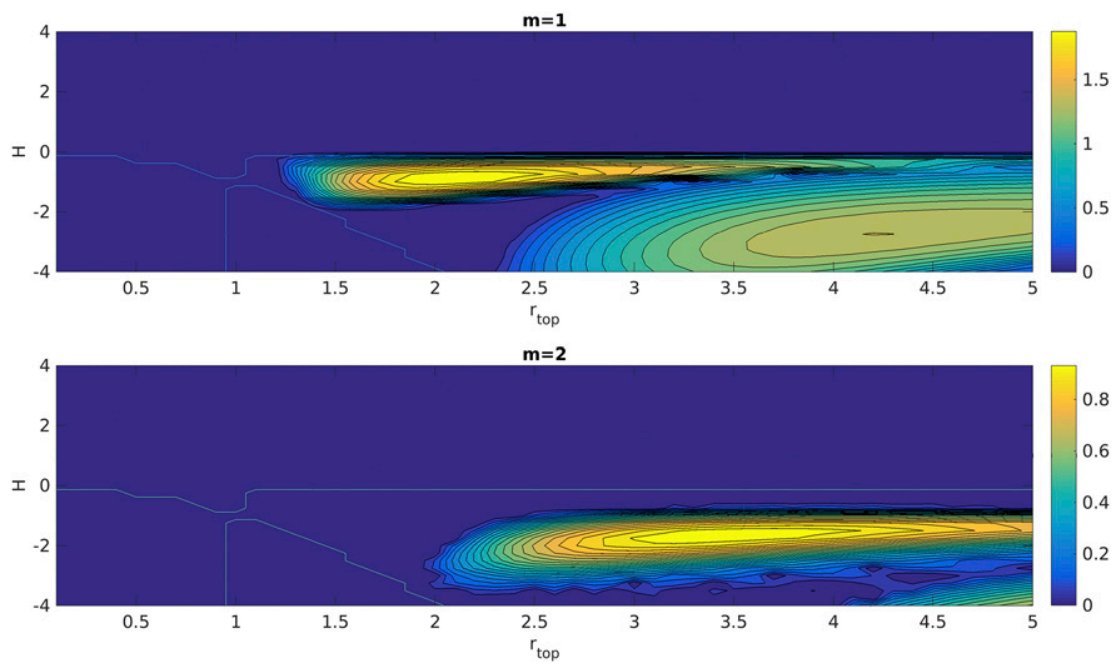

FIG. A1. Growth rate for the baroclinic two-step vortex as a function of topographic size $r_{\text {top }}$ and topographic height $H$. Shown are (top) $m=1$ and (bottom) $m=2$. The single contour line encloses configurations that admit the PV gradient switching sign. Note that $r_{\text {top }}=1$ only admits monotonic PV profiles and hence stable solutions.

linearly unstable vortices to undergo more violent distortions than linear stable vortices, when both approaching the topography from far-field.

The quasigeostrophic approximation assumes small interfacial variations and weak topography. These are not strictly true for energetic vortices and large amplitude topography although numerical studies using primitive equations (e.g., Kamenkovich et al. 1996) agree with our results qualitatively. A previous stability analysis with a two-layer rotating shallow water model revealed significant ageostrophic generalization of standard barotropic instabilities (Lahaye and Zeitlin 2015; note though they scale the length by the barotropic Rossby deformation radius while we normalize the length by the baroclinic Rossby deformation radius thus we are in their large Burger number regime); further investigation with rotating shallow water models (Ribstein and Zeitlin 2013) would be beneficial. Our stability analysis limits to the case with vortex sitting on top of seamounts or depressions while realistic vortextopography encounters involve other processes important for deciding vortex propagation/evolution; for example, Beismann et al. (1999) found formation of along-slope currents by rectification of Rossby waves that in turn advect vortices. Last, we focus on examining a circular vortex while strong ellipticity was shown to excite instability (Nycander and Lacasce 2004). Nonetheless, our idealistic configuration yields general insight into topography effect on vortex stability.

Acknowledgments. BZ and GF would like to acknowledge the support of the GFD Summer School, where the baroclinic results were developed. Support from ENSTA ParisTech helped EC-G study the barotropic problem during her internship at MIT. Support from NASA NESSF program (18-EARTH18F-231) to BZ is acknowledged. Support from the U.S.-Israel Science Foundation (BSF), Grant 2014206 is acknowledged. We are very grateful for conversations with Ziv Kizner when this research began as an offshoot of joint research and also discussions with Ted Johnson at GFD. We also thank the referees for many helpful comments.

\section{APPENDIX}

\section{Linear Stability for Gaussian Vorticity Profiles and Gaussian-Shaped Topography}

As Gaussian vorticity profiles and Gaussian-shaped topography are typically adopted in previous laboratory and analytical studies, we present the linear growth rate for such configurations in the barotropic case.

The topography profile is

$$
h=H \exp \left[-\left(\frac{r}{r_{\text {top }}}\right)^{2}\right],
$$

while the vorticity profile is

$$
\zeta=\zeta_{0} \exp \left[-\left(\frac{r}{r_{0}}\right)^{2}\right] .
$$

The mean velocity profile follows 


$$
V=\frac{\zeta_{0} r_{0}^{2}}{2 r}\left\{1-\exp \left[-\left(\frac{r^{2}}{r_{0}}\right)\right]\right\}
$$

We set $\zeta_{0}=1$ and $r_{0}=1$ while varying topography height $H$ and size $r_{\text {top. }}$. As noted above, by the parity symmetry of QG theory, cyclones with negative signed topography are equivalent to anticyclones with seamounts.

Figure A1 shows that the results are rather similar to Rankin vortex calculations: only oppositely signed, larger scale topography gives instability. On the other hand, the $m=1$ mode could be unstable for the Gaussian vortex, as found for the two-step barotropic vortex but not for the Rankin vortex ( $m=1$ mode is stable).

\section{REFERENCES}

Adams, D. K., and G. R. Flierl, 2010: Modeled interactions of mesoscale eddies with the east Pacific rise: Implications for larval dispersal. Deep-Sea Res. I, 57, 1163-1176, https:// doi.org/10.1016/j.dsr.2010.06.009.

Beismann, J.-O., R. H. Käse, and J. R. Lutjeharms, 1999: On the influence of submarine ridges on translation and stability of agulhas rings. J. Geophys. Res., 104, 7897-7906, https://doi.org/ 10.1029/1998JC900127.

Biebuyck, G. L., 1986: Self propagation of a barotropic circular eddy. Woods Hole Oceanographic Institution Tech. Rep. WHOI-86-45, 193-197.

Bretherton, F. P., and D. B. Haidvogel, 1976: Two-dimensional turbulence above topography. J. Fluid Mech., 78, 129-154, https://doi.org/10.1017/S002211207600236X.

Carnevale, G., R. Kloosterziel, and G. van Heijst, 1991: Propagation of barotropic vortices over topography in a rotating tank. J. Fluid Mech., 233, 119-139, https://doi.org/10.1017/ S0022112091000411.

Flierl, G. R., 1988: On the instability of geostrophic vortices. J. Fluid Mech., 197, 349-388, https://doi.org/10.1017/ S0022112088003283.

Ford, R., M. E. McIntyre, and W. A. Norton, 2000: Balance and the slow quasimanifold: Some explicit results. J. Atmos. Sci., 57, 1236-1254, https://doi.org/10.1175/1520-0469(2000)057<1236: BATSQS $>2.0 . \mathrm{CO} ; 2$.

Garzoli, S. L., P. L. Richardson, C. M. Duncombe Rae, D. M. Fratantoni, G. J. Goñi, and A. J. Roubicek, 1999: Three Agulhas rings observed during the Benguela Current Experiment. J. Geophys. Res., 104, 20 971-20 985, https://doi.org/10.1029/ 1999JC900060.

Goni, G. J., S. L. Garzoli, A. J. Roubicek, D. B. Olson, and O. B. Brown, 1997: Agulhas ring dynamics from TOPEX/POSEIDON satellite altimeter data. J. Mar. Res., 55, 861-883, https://doi.org/ 10.1357/0022240973224175.

Gould, W. J., R. Hendry, and H. E. Huppert, 1981: An abyssal topographic experiment. Deep-Sea Res., 28A, 409-440, https:// doi.org/10.1016/0198-0149(81)90135-7.

Grimshaw, R., X. He, P. Sun, and D. Broutman, 1994a: Analytical and numerical study of a barotropic eddy on a topographic slope. J. Phys. Oceanogr., 24, 1587-1607, https://doi.org/ 10.1175/1520-0485(1994)024<1587:AANSOA>2.0.CO;2.

— , Y. Tang, and D. Broutman, 1994b: The effect of vortex stretching on the evolution of barotropic eddies over a topographic slope. Geophys. Astrophys. Fluid Dyn., 76 , 43-71, https://doi.org/10.1080/03091929408203659.

Hart, J., 1975a: Baroclinic instability over a slope. Part I: Linear theory. J. Phys. Oceanogr., 5, 625-633, https://doi.org/10.1175/ 1520-0485(1975)005<0625:BIOASP $>2.0 . \mathrm{CO} ; 2$.

_ 1975 b: Baroclinic instability over a slope. Part II: Finiteamplitude theory. J. Phys. Oceanogr., 5, 634-641, https://doi.org/ 10.1175/1520-0485(1975)005<0634:BIOASP $>2.0 . C O ; 2$.

Huppert, H. E., and K. Bryan, 1976: Topographically generated eddies. Deep-Sea Res. Oceanogr. Abstr., 23, 655-679, https:// doi.org/10.1016/S0011-7471(76)80013-7.

Johnson, E., 1978: Trapped vortices in rotating flow. J. Fluid Mech., 86, 209-224, https://doi.org/10.1017/S0022112078001093.

Kamenkovich, V. M., Y. P. Leonov, D. A. Nechaev, D. A. Byrne, and A. L. Gordon, 1996: On the influence of bottom topography on the Agulhas eddy. J. Phys. Oceanogr., 26, 892-912, https://doi.org/ 10.1175/1520-0485(1996)026<0892:OTIOBT > 2.0.CO;2.

Kizner, Z., V. Makarov, L. Kamp, and G. van Heijst, 2013: Instabilities of the flow around a cylinder and emission of vortex dipoles. J. Fluid Mech., 730, 419-441, https://doi.org/10.1017/ jfm.2013.345.

LaCasce, J. H., 1998: A geostrophic vortex over a slope. J. Phys. Oceanogr., 28, 2362-2381, https://doi.org/10.1175/15200485(1998)028<2362:AGVOAS > 2.0.CO;2.

Lahaye, N., and V. Zeitlin, 2015: Centrifugal, barotropic and baroclinic instabilities of isolated ageostrophic anticyclones in the two-layer rotating shallow water model and their nonlinear saturation. J. Fluid Mech., 762, 5-34, https://doi.org/10.1017/jfm.2014.631.

— clones and their evolution with a moist convective rotating shallow-water model. J. Atmos. Sci., 73, 505-523, https:// doi.org/10.1175/JAS-D-15-0115.1.

McDonagh, E. L., K. J. Heywood, and M. P. Meredith, 1999: On the structure, paths, and fluxes associated with agulhas rings. J. Geophys. Res., 104, 21 007-21 020, https://doi.org/10.1029/ 1998JC900131.

Meacham, S., 1991: Meander evolution on piecewise-uniform, quasigeostrophic jets. J. Phys. Oceanogr., 21, 1139-1170, https://doi.org/ 10.1175/1520-0485(1991)021<1139:MEOPUQ > 2.0.CO;2.

Nycander, J., and J. Lacasce, 2004: Stable and unstable vortices attached to seamounts. J. Fluid Mech., 507, 71-94, https:// doi.org/10.1017/S0022112004008730.

Olson, D. B., 1980: The physical oceanography of two rings observed by the cyclonic ring experiment. Part II: Dynamics. J. Phys. Oceanogr., 10, 514-528, https://doi.org/10.1175/15200485(1980)010<0514:TPOOTR >2.0.CO;2.

- 1991: Rings in the ocean. Annu. Rev. Earth Planet. Sci., 19, 283-311, https://doi.org/10.1146/annurev.ea.19.050191.001435.

_ , and R. H. Evans, 1986: Rings of the Agulhas current. Deep-Sea Res., 33A, 27-42, https://doi.org/10.1016/0198-0149(86)90106-8.

Polvani, L., N. Zabusky, and G. Flierl, 1988: Applications of contour dynamics to two layer quasigeostrophic flows. Fluid Dyn. Res., 3, 422-424, https://doi.org/10.1016/0169-5983(88)90103-7.

Pullin, D., 1992: Contour dynamics methods. Annu. Rev. Fluid Mech., 24, 89-115, https://doi.org/10.1146/annurev.fl.24.010192.000513.

Rabinovich, M., Z. Kizner, and G. Flierl, 2018: Bottom-topography effect on the instability of flows around a circular island. J. Fluid Mech., 856, 202-227, https://doi.org/10.1017/jfm.2018.705.

Ribstein, B., and V. Zeitlin, 2013: Instabilities of coupled density fronts and their nonlinear evolution in the two-layer rotating shallow-water model: Influence of the lower layer and of the topography. J. Fluid Mech., 716, 528-565, https://doi.org/ 10.1017/jfm.2012.556. 
Richardson, P. L., 1981: Anticyclonic eddies generated near the corner rise seamounts. Tech. Rep. WHOI-81-20, Woods Hole Oceanographic Institution, 20 pp., https://oi.org/10.1575/1912/10240.

Schmidt, G., and E. Johnson, 1997: Instability in stratified rotating shear flow along ridges. J. Mar. Res., 55, 915-933, https:// doi.org/10.1357/0022240973224201.

Schouten, M. W., W. P. Ruijter, P. J. Leeuwen, and J. R. Lutjeharms, 2000: Translation, decay and splitting of agulhas rings in the southeastern atlantic ocean. J. Geophys. Res., 105, 21 913-21 925, https://doi.org/10.1029/1999JC000046.

Smith, D. C., and J. O'Brien, 1983: The interaction of a two-layer isolated mesoscale eddy with bottom topography. J. Phys. Oceanogr., 13, 1681-1697, https://doi.org/10.1175/1520-0485(1983) 013<1681:TIOATL > 2.0.CO;2.

Stern, M. E., 1987: Horizontal entrainment and detrainment in largescale eddies. J. Phys. Oceanogr., 17, 1688-1695, https://doi.org/ 10.1175/1520-0485(1987)017<1688:HEADIL>2.0.CO;2.

— vortex. J. Phys. Oceanogr., 28, 22-39, https://doi.org/10.1175/ 1520-0485(1998)028<0022:TSPQMV>2.0.CO;2.

van Aken, H., A. van Veldhoven, C. Veth, W. De Ruijter, P. van Leeuwen, S. Drijfhout, C. Whittle, and M. Rouault, 2003: Observations of a young Agulhas ring, Astrid, during MARE in March 2000. Deep-Sea Res. II, 50, 167-195, https://doi.org/ 10.1016/S0967-0645(02)00383-1. van Geffen, J., and P. Davies, 2000: A monopolar vortex encounters a north-south ridge or trough. Fluid Dyn. Res., 26, 157-179, https://doi.org/10.1016/S0169-5983(99)00022-2.

Verron, J., and C. Le Provost, 1985: A numerical study of quasi-geostrophic flow over isolated topography. J. Fluid Mech., 154, 231-252, https://doi.org/10.1017/ S0022112085001501.

Wang, X., 1992: Interaction of an eddy with a continental slope. Ph.D. thesis, Massachusetts Institute of Technology, 216 pp., https://doi.org/10.1575/1912/5490.

Wei, J., D.-P. Wang, and C. N. Flagg, 2008: Mapping gulf stream warm core rings from shipboard ADCP transects of the Oleander Project. J. Geophys. Res., 113, C10021, https:// doi.org/10.1029/2007JC004694.

Zabusky, N. J., M. Hughes, and K. Roberts, 1979: Contour dynamics for the euler equations in two dimensions. J. Comput. Phys., 30, 96-106, https://doi.org/10.1016/00219991(79)90089-5.

Zavala Sansón, L., 2002: Vortex-ridge interaction in a rotating fluid. Dyn. Atmos. Oceans, 35, 299-325, https://doi.org/10.1016/ S0377-0265(02)00014-3.

, A. B. Aguiar, and G. van Heijst, 2012: Horizontal and vertical motions of barotropic vortices over a submarine mountain. J. Fluid Mech., 695, 173-198, https://doi.org/10.1017/ jfm.2012.9. 